\title{
A POD-Galerkin Reduced-Order Model for Isotropic Viscoelastic
}

\author{
Turbulent Flow
}

\author{
Jingjing Chen ${ }^{1}$, Dongxu $\mathrm{Han}^{2}$, Bo Yu ${ }^{2 *}$, Dongliang $\mathrm{Sun}^{2}$, Jinjia Wei ${ }^{3}$
}

1. National Engineering Laboratory for Pipeline Safety , Beijing Key Laboratory of Urban Oil and

Gas Distribution Technology, China University of Petroleum, Beijing 102249, China

2. School of Mechanical Engineering, Beijing Institute of Petrochemical Technology, Beijing

102617, China

3. State Key Laboratory of Multiphase Flow in Power Engineering, Xi'an Jiaotong University, Xi'an 710049, China

*Correspondent author: yubobox@vip.163.com

\begin{abstract}
In this article, a proper orthogonal decomposition (POD) reduced-order model for isotropic turbulent flow of viscoelastic fluid is established for the first time. Particularly, since the present studies about viscoleastic fluid are mainly for revealing the mechanism of turbulence, we try to establish the reduced order model for momentum equations and constitutive equations, finally get both velocities and deformation rates calculated. Through decomposing the sampling matrices which are obtained by direct numerical simulation (DNS) using finite volume method (FVM), the velocity basis functions and deformation rate basis functions are generated respectively. According to the Galerkin projection method, the equations for velocity spectrum coefficients and deformation rate spectrum coefficients are deducted, which are coupled pluralistic nonlinear equations and solved iteratively by the Newton-Raphson method. To illustrate the performance of the proposed model for the viscoelastic fluid flow, a two-dimensional decaying isotropic turbulence testing case is designed in Example 1. It is found that the established reduced-order model obtains good accuracy when the decaying flow is at its early stage, but the errors get considerable when the flow steps into transition flow. In addition, a three-dimensional forced isotropic viscoelastic turbulence testing case is designed in Example 2. It is indicated that the errors of viscoelastic forced isotropic turbulent flow are acceptable. Finally, the calculation speed of the established reduced-order model is found to be much faster than that of DNS.
\end{abstract}

Keywords: Proper orthogonal decomposition; Galerkin projection; Decaying isotropic turbulent flow; Forced isotropic turbulent flow; Viscoelastic fluid.

\section{Nomenclature}

$a, b$ spectral coefficients 
C deformation rate

$\bar{C}$ mean deformation rate

$C^{\prime}$ fluctuation of deformation rate

$e$ velocity error, $e=\left(\sum_{l=1}^{N_{\text {tof }}}\left|u_{P O D}(l)-u_{D N S}(l)\right|\right) /\left(\sum_{l=1}^{N_{\text {tor }}}\left|u_{D N S}(l)\right|\right)$

$f(r)$ Peterlin function

$L$ maximum tensile elongation of the viscoelastic fluid molecules, $\mathrm{m}$

$M$ total number of the basis functions

$N$ total sampling number

$N_{\text {tot }}$ total test number

$p$ pressure, $\mathrm{Pa}$

$r$ length of the viscoelastic fluid molecules, $\mathrm{m}$

$T^{[s]}$ viscous stress induced by solvent, $\mathrm{Pa}$

$T^{[p]}$ viscoelastic stress of the viscoelastic fluid, $\mathrm{Pa}$

$\boldsymbol{u}$ velocity vector, $\mathrm{m} / \mathrm{s}$

$\bar{u}$ mean velocity vector, $\mathrm{m} / \mathrm{s}$

u' fluctuation of velocity, $\mathrm{m} / \mathrm{s}$

$T 1_{k i}, A 1_{k i}, B 1_{k i}, D 1_{k i}, C 1_{i}$ coefficients in the momentum reduced model

$T 2_{k i}, A 2_{k l i}, B 2_{k i}, D 2_{k i}, C 2_{i}$ coefficients in the constitutive reduced model

\section{Greek symbols}

$\rho$ density, $\mathrm{kg} / \mathrm{m}^{3}$

$\tau_{p}$ relaxation time, $\mathrm{s}$

$\Gamma_{i j}$ velocity gradient, $1 / \mathrm{s}$

$\mu$ dynamic viscosity, $\mathrm{Pa} \cdot \mathrm{s}$

$\alpha$ liquidity factor

$v^{[p]}$ kinematic viscosity of the viscoelastic fluid, $\mathrm{m}^{2} / \mathrm{s}$

$v^{[s]}$ kinematic viscosity of the solvent, $\mathrm{m}^{2} / \mathrm{s}$

$\beta$ measurement of viscoelastic fluid solution concentrationration, $\beta=\frac{v^{[s]}}{v^{[s]}+v^{[p]}}$

$\phi$ velocity basis function, $\mathrm{m} / \mathrm{s}$

$\varphi$ deformation rate basis function

$\lambda$ eigenvalue

$\varsigma$ energy contribution 


\section{Introduction}

Turbulence is the most common flow phenomena and is the last great unsolved problem of classical physics [1]. Direct numerical simulation (DNS) is resolving all the scales present in a turbulent flow without any modeling, but it is not a realistic possibility in most cases of practical importance. In the foreseeable future, the cost of such simulation will remain far beyond people's means, and will be limited to low Reynolds numbers and simple geometries.

Proper orthogonal decomposition (POD) can extract characteristic information (basis functions) from large amount of data obtained by DNS and the basis functions can be used to depict turbulence efficiently.

POD was firstly introduced to the turbulence by Lumley in 1967 [2]. In the past 60 years, it was mainly used in two aspects of turbulence study: turbulence analysis and reduced order model study.

For the fundamental mechanisms of turbulence study, early applications of the POD were reported by Bakewell et al. [3] who applied the POD to a turbulent pipe flow. Then the POD was applied to a lot of different flow patterns and geometries. The basis functions in the wall region of a turbulent boundary layer were computed by Herzog [4]. Rempfer [5, 6] also computed the POD basis functions of a turbulent boundary layer. Then, they used basis functions to study the flow structures to understand the turbulence. W. Cazemier [20] investigated the turbulent flows carefully and studied the structures based on basis functions. Snapshot proper orthogonal decomposition (POD) was utilized to understand the turbulent channel flow physics over permeable porous surfaces [21]. Their aim was to study how the flow structures vary in different wall permeabilities. The study [22] investigated the energies and scales associated with turbulent flow features and how they are influenced by wall heating. The POD analysis and spectral analysis provided information about the flow energy and its patterns at different modes and gave a deep insight into the scales and energies of the physical flow structures.

In reduced order model research, Aubry et al. [7] used the POD basis functions of Herzog [4] to derive a low-dimensional dynamical system for the wall region of a turbulent boundary layer and found that the pressure signal of the outer part of the boundary layer triggers the bursting events associated with stream-wise vortex pairs. Holmes et al. [8] focused on low wave number phenomena of turbulence generation, appealing to simple and conventional modeling of inertial range transport and energy dissipation. Johansson et al. [9] found that the reduced dynamical systems are able to reproduce the mean velocity and Reynolds stress profiles as long as the aspect ratio of the channel or the Reynolds number does not increase or decrease from the original setting 
by more than $50 \%$ or $30 \%$, respectively. Peter S. Johansson et al. [10] used the method to test on a DNS of $\mathrm{R}_{*}=180$ and a LES of $\mathrm{R}_{*}=400$. Statistics such as mean velocity, rms-profiles, and turbulent shear-stress become close to the fully developed state within 1500 wall units downstream the inlet. The POD method was used for the first time to study the convective flow in a simulated Czochralski system [11]. B. Podvin [12] investigated a POD-based model for the wall layer of a turbulent channel flow. W. Cazemier [17] established reduced order model for cavity flow.

POD reduced order model for turbulence has been studied a lot, however, only few researches are about POD reduced order model for viscoelastic fluid turbulence. Gaurab Samanta et al. [15] studied the effect of $\mathrm{K}-\mathrm{L}$ data reduction on velocity and conformation statistics in a viscoelastic turbulent channel flow calculated with the Giesekus model under conditions for which $37.4 \%$ drag reduction has been obtained. It was found to increase with viscoelsticity that was well reproduced in the reduced K-L data except near the channel centerline where the K-L data showed some loss of anisotropy. Later, they investigated three different data reduction approaches in order to introduce small-scale information [16]. It is shown that the conformation field can be reconstructed fairly accurately using the K-L decomposition. In addition, many efforts have been focused on the wall-bounded turbulent flows where the wall plays an important role, such as channel, pipe and boundary layer flows. The existence of the wall makes the flow inhomogeneous, so it is hard to investigate the interaction between the micro-molecular structures of viscoelastic fluid and turbulent vortex structures. However, the study on isotropic turbulence in viscoelastic fluid can solve well the above problems. This is because there is no mean shear stress in the flow; the existence of viscoelastic fluid cannot change the momentum transportation of flow system, but it can change the turbulent kinetic energy cascading from large-scale to small-scale. So the study of isotropic turbulence in viscoelastic fluid has important theoretical meaning and scientific value. The two most frequently studied types of isotropic turbulence are freely decaying and forced (statistically stationary) turbulence. V.M. Tikhomirov [14] did some research on the isotropic turbulent flow about the Newtonian fluid. Kourentis [18] studied forced turbulent wakes by combining particle image velocimetry (PIV), POD and finite-time Lyapunov exponent (FTLE) and uncovered large-scale coherent structures successfully. Orellano and Wengle [19] analyzed coherent structures in forced turbulent flow over a fence and found the successive features of the generation, breakup, and shedding of the vortex originated from the rear pulsating jet. This vortex shedding from the separation bubble is known to be the mechanism of reduction of the time-mean length of the separation bubble by the front pulsating jet control. Stein David et al [23] used proper orthogonal decomposition (POD) to extract the dominant oscillatory flow features in an effort to understand the elastic instability and the possible transition to elastic turbulence.

The above studies for viscoelastic isotropic turbulence are for statistics analysis and revealing 
mechanisms. However, fast calculations of complete velocity field for isotropic turbulence with different flow parameters are always required. In this article, our main goal is to calculate the velocity field with fewer, more efficient modes (basis functions) by applying the POD method to the isotropic turbulent flow. Particularly, for viscoelastic fluid turbulent flow, the above studies have not established reduced-order model of deformation equations and then computed the deformation rates. However, we focus on the point and realized the establishment of POD reduced order model of deformation equations, and finally got both deformation rates and velocity fields solved successfully.

This paper is organized as follows. Firstly, the methodology of turbulent flow based on the POD reduced-order model for viscoelastic fluid is introduced in section 2. Next, the viscoelastic decaying isotropic turbulent flow and forced isotropic turbulence examples are presented to illustrate the performance of the proposed model in section 3. Then, some conclusions are given in section 4.

\section{Methodology}

In this section, method about the solving of fluid turbulent flow is first descripting carefully. The main lines are: (1) governing equations for the viscoelastic fluid turbulent flow are first introduced. (2) The principle of basis functions calculation is given briefly. (3) The establishment of the proposed POD-Galerkin reduced-order model is presented in detail.

\subsection{Governing equations for viscoelastic fluid turbulent flow}

A viscoelastic fluid turbulent flow problem, is considered herein, which is governed by momentum equation and the deformation equation. The turbulent flow can be depicted by the mass conservation equation (1), momentum conservation equation (2) and general deformation equation (3).

$$
\begin{gathered}
\nabla \cdot \boldsymbol{u}=0 \\
\frac{\partial \boldsymbol{u}}{\partial t}+\boldsymbol{u} \cdot \nabla \boldsymbol{u}=-\frac{1}{\rho} \nabla p+\frac{1}{\rho} \nabla \cdot \boldsymbol{T}^{[s]}+\frac{1}{\rho} \nabla \cdot \boldsymbol{T}^{[p]} \\
\frac{\partial \boldsymbol{C}}{\partial t}+\boldsymbol{u} \cdot \nabla \boldsymbol{C}=\boldsymbol{C} \cdot \nabla \boldsymbol{u}+\nabla \boldsymbol{u}^{\mathrm{T}} \cdot \boldsymbol{C}-\frac{\mathbf{1}}{\tau_{p}}[\mathbf{I}+\alpha(\boldsymbol{C}-\mathbf{I})] \cdot[f(r) \boldsymbol{C}-\mathbf{I}]
\end{gathered}
$$

Where, $\boldsymbol{u}$ is the dimensional velocity; $\boldsymbol{C}$ is the deformation rate of the viscoelastic fluid molecules; $\tau_{p}$ is the relaxation time of viscoelastic fluid, and $\alpha$ is the liquidity factor.

According to the viscous stress constitutive model of Newtonian fluid [15], the viscous stress induced by solvent $\boldsymbol{T}^{[s]}$ is:

$$
\boldsymbol{T}^{[s]}=\rho v^{[s]}\left(\Gamma_{i j}+\Gamma_{j i}\right)
$$


Where $v^{[s]}$ is the kinematic viscosity of the solvent; $\Gamma_{i j}$ is the gradient of the velocity. The viscoelastic stress of the viscoelastic fluid $\boldsymbol{T}^{[p]}$ which is depicted by the dumbbell model [15]:

$$
\boldsymbol{T}^{[p]}=\frac{\rho v^{[p]}}{\tau_{p}}(f(r) \boldsymbol{C}-\mathbf{I})
$$

Where, $v^{[p]}$ is the kinematic viscosity of the viscoelastic fluid, $r$ is the length of the viscoelastic fluid molecules, determined by $r=\sqrt{\operatorname{trace}(\boldsymbol{C})}$. The term $f(r)$ denotes the Peterlin function [16].

In the proposed FENE-P model [16]

$$
\begin{gathered}
\alpha=0 \\
f(r)=\left(L^{2}-3\right) /\left(L^{2}-r^{2}\right)
\end{gathered}
$$

Where, $L$ is the maximum tensile elongation of the viscoelastic fluid molecules.

Finally, we get the governing equations in detail as follows [16]:

$$
\begin{gathered}
\nabla \cdot \boldsymbol{u}=0 \\
\frac{\partial \boldsymbol{u}}{\partial t}+\boldsymbol{u} \cdot \nabla \boldsymbol{u}=-\frac{1}{\rho} \nabla p+\frac{\mu}{\rho} \Delta \boldsymbol{u}+\frac{\beta \mu}{\tau_{p}} \cdot \frac{L^{2}-3}{L^{2}-r^{2}} \nabla \cdot \boldsymbol{C} \\
\frac{\partial \boldsymbol{C}}{\partial t}+\boldsymbol{u} \cdot \nabla \boldsymbol{C}=\boldsymbol{C} \cdot \nabla \boldsymbol{u}+\nabla \boldsymbol{u}^{\mathrm{T}} \cdot \boldsymbol{C}-\frac{1}{\tau_{p}} \cdot\left[\frac{L^{2}-3}{L^{2}-r^{2}} \boldsymbol{C}-\mathbf{I}\right]
\end{gathered}
$$

\subsection{Calculation of basis functions}

For viscoelastic fluid flow, two types of basis functions are included which are velocity basis functions and deformation rate basis functions and they can be obtained by decomposing corresponding sampling matrices using 'snapshots method' [13].Suppose that velocity fields and deformation rate fields in $N$ time instants are obtained by DNS on a mesh system with $p^{\prime} q^{\prime} l$ grids, the specific processes are as follows:

First, both velocity and deformation rate obtained by DNS are written as the summation of time mean field $\bar{u}(\bar{C})$ and fluctuations $\boldsymbol{u}^{\prime}\left(\boldsymbol{C}^{\prime}\right)$

$$
\begin{aligned}
& \boldsymbol{u}=\overline{\boldsymbol{u}}+\boldsymbol{u}^{\prime} \\
& \boldsymbol{C}=\overline{\boldsymbol{C}}+\boldsymbol{C}^{\prime}
\end{aligned}
$$

Where $\overline{\boldsymbol{u}}$, the time mean velocity, is determined by equation (12), and $\overline{\boldsymbol{C}}$ is determined by equation (13)

$$
\begin{aligned}
\overline{\boldsymbol{u}} & =\frac{1}{T} \int_{t_{0}}^{t_{0}+T} \boldsymbol{u} d t \\
\overline{\boldsymbol{C}} & =\frac{1}{T} \int_{t_{0}}^{t_{0}+T} \boldsymbol{C} d t
\end{aligned}
$$


Subsequently, the fluctuation fields of velocity and deformation rate are used to construct corresponding sampling matrices which can be seen in Fig. 1

Finally, 'snapshot method' is employed to decompose matrices shown in Fig.1 and the basis functions for velocity and deforming rate can be obtained.

According to the properties of POD basis functions, $\boldsymbol{u}^{\prime}$ and $\boldsymbol{C}^{\prime}$ can be expanded in basis functions as follows:

$$
\begin{aligned}
& \boldsymbol{u}^{\prime}=\sum_{k=1}^{\infty} a_{k} \boldsymbol{\phi}_{k} \\
& \boldsymbol{C}^{\prime}=\sum_{k=1}^{\infty} b_{k} \boldsymbol{\varphi}_{k}
\end{aligned}
$$

Where $\phi_{k}$ and $\varphi_{k}$ are the velocity basis functions and deformation rate basis functions, respectively.

\subsection{Establishment of the reduced-order model}

As it is shown in Eq. (1) (3), the governing equation for the viscoelastic turbulent flow consists of mass conservation equation, momentum conservation equation and general deformation equation. As we all know, the velocity field generated by the combination of basis functions can satisfy the conservation equation automatically [13]. So, just reduced-order models for momentum equation and general deformation equation are needed to be established.

(1) Reduced-order model of the momentum equation

Substituting Eqs.(10), (11) into Eq. (8), gives

$$
\frac{\partial \boldsymbol{u}^{\prime}}{\partial t}+\overline{\boldsymbol{u}} \cdot \nabla \overline{\boldsymbol{u}}+\overline{\boldsymbol{u}} \cdot \nabla \boldsymbol{u}^{\prime}+\boldsymbol{u}^{\prime} \cdot \nabla \overline{\boldsymbol{u}}+\boldsymbol{u}^{\prime} \cdot \nabla \boldsymbol{u}^{\prime}=-\frac{1}{\rho} \nabla p+\frac{\mu}{\rho} \Delta \overline{\boldsymbol{u}}+\frac{\mu}{\rho} \Delta \boldsymbol{u}^{\prime}+\left(\frac{\beta \mu}{\tau_{p}} \cdot \frac{L^{2}-3}{L^{2}-r^{2}}\right) \nabla \cdot \overline{\boldsymbol{C}}+\left(\frac{\beta \mu}{\tau_{p}} \cdot \frac{L^{2}-3}{L^{2}-r^{2}}\right) \nabla \cdot \boldsymbol{C}^{\prime}
$$

Substituting Eqs.(14), (15) into Eq. (16), we obtain

$$
\begin{aligned}
& \sum_{k=1}^{M} \frac{d a_{k}}{d t} \boldsymbol{\phi}_{k}+\overline{\boldsymbol{u}} \cdot \nabla \overline{\boldsymbol{u}}+\sum_{k=1}^{M} a_{k} \overline{\boldsymbol{u}} \cdot \nabla \boldsymbol{\phi}_{k}+\sum_{k=1}^{M} a_{k} \boldsymbol{\phi}_{k} \cdot \nabla \overline{\boldsymbol{u}}+\sum_{k=1}^{M} \sum_{l=1}^{M} a_{k} a_{l} \boldsymbol{\phi}_{k} \cdot \nabla \boldsymbol{\phi} \\
& =-\frac{1}{\rho} \nabla p+\frac{\mu}{\rho} \Delta \overline{\boldsymbol{u}}+\sum_{k=1}^{M} a_{k} \frac{\mu}{\rho} \Delta \boldsymbol{\phi}_{k}+\left(\frac{\beta \mu}{\tau_{p}} \cdot \frac{L^{2}-3}{L^{2}-r^{2}}\right) \nabla \cdot \overline{\boldsymbol{C}}+\sum_{k=1}^{M} b_{k}\left(\frac{\beta \mu}{\tau_{p}} \cdot \frac{L^{2}-3}{L^{2}-r^{2}}\right) \nabla \cdot \boldsymbol{\varphi}_{k}
\end{aligned}
$$

Where " $M$ " is the total number of basis functions we choose in the reduced-order model.

Projecting Eq. (17) onto the space of velocity basis function $\phi_{l}$, yields

$$
\begin{aligned}
& \sum_{k=1}^{M} \frac{d a_{k}}{d t}\left(\boldsymbol{\phi}_{k}, \boldsymbol{\phi}_{l}\right)+\left(\overline{\boldsymbol{u}} \cdot \nabla \overline{\boldsymbol{u}}, \boldsymbol{\phi}_{i}\right)+\sum_{k=1}^{M} a_{k}\left(\overline{\boldsymbol{u}} \cdot \nabla \boldsymbol{\phi}_{k}, \boldsymbol{\phi}_{l}\right)+\sum_{k=1}^{M} a_{k}\left(\boldsymbol{\phi}_{k} \cdot \nabla \overline{\boldsymbol{u}}, \boldsymbol{\phi}_{i}\right)+\sum_{k=1}^{M} \sum_{l=1}^{M} a_{k} a_{l}\left(\boldsymbol{\phi}_{k} \cdot \nabla \boldsymbol{\phi}, \boldsymbol{\phi}_{l}\right) \\
& =-\left(\frac{1}{\rho} \nabla p, \boldsymbol{\phi}_{i}\right)+\left(\frac{\mu}{\rho} \Delta \overline{\boldsymbol{u}}, \boldsymbol{\phi}_{l}\right)+\sum_{k=1}^{M} a_{k}\left(\frac{\mu}{\rho} \Delta \boldsymbol{\phi}_{k}, \boldsymbol{\phi}_{i}\right)+\left(\left(\frac{\beta \mu}{\tau_{p}} \cdot \frac{L^{2}-3}{L^{2}-r^{2}}\right) \nabla \cdot \overline{\boldsymbol{C}}, \boldsymbol{\phi}_{i}\right)+\sum_{k=1}^{M} b_{k}\left(\left(\frac{\beta \mu}{\tau_{p}} \cdot \frac{L^{2}-3}{L^{2}-r^{2}}\right) \nabla \cdot \boldsymbol{\phi}_{k}, \boldsymbol{\phi}_{l}\right)
\end{aligned}
$$


The pressure term $-\frac{1}{\rho}\left(\nabla p, \phi_{i}\right)$ is zero. Gauss theorem states that

$$
-\frac{1}{\rho}\left(\nabla p, \phi_{i}\right)=-\frac{1}{\rho}\left[-\left(\operatorname{div} \phi_{i}, p\right)+\int_{\Omega} \phi_{i} \cdot n p\right]
$$

Both terms on the right-hand are zero since the POD velocity basis functions are divergence-free and satisfy the homogeneous boundary conditions.

Thus the POD-Galerkin reduced-order model of momentum equation is:

$$
\sum_{k=1}^{M} \frac{d a_{k}}{d t} T 1_{k i}+\sum_{k=1}^{M} \sum_{k=1}^{M} a_{k} a_{l} A 1_{k l i}+\sum_{k=1}^{M} a_{k} B 1_{k i}+\sum b_{k} D 1_{k i}=C 1_{i}
$$

Where

$$
\begin{gathered}
T 1_{k i}=\left(\boldsymbol{\phi}_{k}, \boldsymbol{\phi}_{i}\right) \\
A 1_{k l i}=\left(\boldsymbol{\phi}_{k} \cdot \nabla \boldsymbol{\phi}_{i}, \boldsymbol{\phi}_{i}\right) \\
B 1_{k i}=\left(\overline{\boldsymbol{u}} \cdot \nabla \boldsymbol{\phi}_{k}, \boldsymbol{\phi}_{i}\right)+\left(\boldsymbol{\phi}_{k} \cdot \nabla \overline{\boldsymbol{u}}, \boldsymbol{\phi}_{i}\right)-\left(\frac{\mu}{\rho} \Delta \boldsymbol{\phi}_{k}, \boldsymbol{\phi}_{i}\right) \\
C 1_{i}=\left(\frac{\mu}{\rho} \Delta \overline{\boldsymbol{u}}, \boldsymbol{\phi}_{i}\right)+\left(\left(\frac{\beta \mu}{\tau_{p}} \cdot \frac{L^{2}-3}{L^{2}-r^{2}}\right) \nabla \cdot \overline{\boldsymbol{C}}, \boldsymbol{\phi}_{i}\right)-\left(\overline{\boldsymbol{u}} \cdot \nabla \overline{\boldsymbol{u}}, \boldsymbol{\phi}_{i}\right) \\
D 1_{k i}=-\left(\left(\frac{\beta \mu}{\tau_{p}} \cdot \frac{L^{2}-3}{L^{2}-r^{2}}\right) \nabla \cdot \boldsymbol{\varphi}_{k}, \boldsymbol{\phi}_{i}\right)
\end{gathered}
$$

(2) Reduced-order model of constitutive equation

Substituting Eqs.(10), (11) into Eq. (9), we obtain

$$
\begin{aligned}
& \frac{\partial \boldsymbol{C}^{\prime}}{\partial t}+\overline{\boldsymbol{u}} \nabla \overline{\boldsymbol{C}}+\overline{\boldsymbol{u}} \nabla \boldsymbol{C}^{\prime}+\boldsymbol{u}^{\prime} \nabla \overline{\boldsymbol{C}}+\boldsymbol{u}^{\prime} \nabla \boldsymbol{C}^{\prime} \\
& =\overline{\boldsymbol{C}} \nabla \overline{\boldsymbol{u}}+\overline{\boldsymbol{C}} \nabla \boldsymbol{u}^{\prime}+\boldsymbol{C}^{\prime} \nabla \overline{\boldsymbol{u}}+\boldsymbol{C}^{\prime} \nabla \boldsymbol{u}^{\prime}+\nabla \overline{\boldsymbol{u}}^{-\mathrm{T}} \overline{\boldsymbol{C}}+\nabla \overline{\boldsymbol{u}}^{\mathrm{T}} \boldsymbol{C}^{\prime}+\nabla \boldsymbol{u}^{\mathrm{T}} \overline{\boldsymbol{C}}+\nabla \boldsymbol{u}^{\mathrm{T}} \boldsymbol{C}^{\prime}+\frac{1}{\tau_{p}} \mathbf{I}-\frac{1}{\tau_{p}} \frac{L^{2}-3}{L^{2}-r^{2}} \overline{\boldsymbol{C}}-\frac{1}{\tau_{p}} \frac{L^{2}-3}{L^{2}-r^{2}} \boldsymbol{C}^{\prime}
\end{aligned}
$$

Substituting Eqs.(14), (15) into Eq. (21), gives

$$
\begin{aligned}
& \sum_{k=1}^{M} \frac{\partial b_{k}}{\partial t} \boldsymbol{\varphi}_{k}+\overline{\boldsymbol{u}} \nabla \overline{\boldsymbol{C}}+\sum_{k=1}^{M} b_{k} \overline{\boldsymbol{u}} \nabla \boldsymbol{\varphi}_{k}+\sum_{k=1}^{M} a_{k} \boldsymbol{\phi}_{k} \nabla \overline{\boldsymbol{C}}+\sum_{k=1}^{M} \sum_{l=1}^{M} a_{k} b_{l} \boldsymbol{\phi}_{k} \nabla \boldsymbol{\varphi}_{l} \\
& =\overline{\boldsymbol{C}} \nabla \overline{\boldsymbol{u}}+\sum_{k=1}^{M} a_{k} \overline{\boldsymbol{C}} \nabla \boldsymbol{\phi}_{k}+\sum_{k=1}^{M} b_{k} \boldsymbol{\varphi}_{k} \nabla \overline{\boldsymbol{u}}+\sum_{k=1}^{M} \sum_{l=1}^{M} a_{k} b_{l} \nabla \boldsymbol{\phi}_{k} \boldsymbol{\varphi}_{l}+\nabla \overline{\boldsymbol{u}} \overline{\boldsymbol{C}}+\sum_{k=1}^{M} b_{k} \nabla \overline{\boldsymbol{u}} \boldsymbol{\varphi}_{k} \\
& +\nabla \sum_{k=1}^{M} a_{k} \boldsymbol{\phi}_{k}^{\mathrm{T}} \overline{\boldsymbol{C}}+\sum_{k=1}^{M} \sum_{l=1}^{M} a_{k} b_{l} \nabla \boldsymbol{\phi}_{k}^{\mathrm{T}} \boldsymbol{\varphi}_{l}+\frac{1}{\tau_{p}} \mathbf{I}-\frac{1}{\tau_{p}} \frac{L^{2}-3}{L^{2}-r^{2}} \overline{\boldsymbol{C}}-\sum_{k=1}^{M} b_{k} \frac{1}{\tau_{p}} \frac{L^{2}-3}{L^{2}-r^{2}} \boldsymbol{\varphi}_{k}
\end{aligned}
$$

Projecting Eq. (22) onto the space of deformation rate basis functions $\varphi_{i}$, yields 


$$
\begin{aligned}
& \sum_{k=1}^{M} \frac{\partial b_{k}}{\partial t}\left(\boldsymbol{\varphi}_{k}, \boldsymbol{\varphi}_{i}\right)+\left(\overline{\boldsymbol{u}} \nabla \overline{\boldsymbol{C}}, \boldsymbol{\varphi}_{i}\right)+\sum_{k=1}^{M} b_{k}\left(\overline{\boldsymbol{u}} \nabla \boldsymbol{\varphi}_{k}, \boldsymbol{\varphi}_{i}\right)+\sum_{k=1}^{M} a_{k}\left(\boldsymbol{\phi}_{k} \nabla \overline{\boldsymbol{C}}, \boldsymbol{\varphi}_{i}\right)+\sum_{k=1}^{M} \sum_{l=1}^{M} a_{k} b_{l}\left(\boldsymbol{\phi}_{k} \nabla \boldsymbol{\varphi}_{l}, \boldsymbol{\varphi}_{i}\right) \\
& =\left(\overline{\boldsymbol{C}} \nabla \overline{\boldsymbol{u}}, \boldsymbol{\varphi}_{i}\right)+\sum_{k=1}^{M} a_{k}\left(\overline{\boldsymbol{C}} \nabla \boldsymbol{\phi}_{k}, \boldsymbol{\varphi}_{i}\right)+\sum_{k=1}^{M} b_{k}\left(\boldsymbol{\varphi}_{k} \nabla \overline{\boldsymbol{u}}, \boldsymbol{\varphi}_{i}\right)+\sum_{k=1}^{M} \sum_{l=1}^{M} a_{k} b_{l}\left(\nabla \boldsymbol{\phi}_{k} \boldsymbol{\varphi}_{l}, \boldsymbol{\varphi}_{i}\right)+\left(\nabla \overline{\boldsymbol{u}} \overline{\boldsymbol{C}}, \boldsymbol{\varphi}_{i}\right)+\sum_{k=1}^{M} b_{k}\left(\nabla \overline{\boldsymbol{u}}^{\mathrm{T}} \boldsymbol{\varphi}_{k}, \boldsymbol{\varphi}_{i}\right) \\
& +\sum_{k=1}^{M} a_{k}\left(\nabla \boldsymbol{\phi}_{k}^{\mathrm{T}} \overline{\boldsymbol{C}}, \boldsymbol{\varphi}_{i}\right)+\sum_{k=1}^{M} \sum_{l=1}^{M} a_{k} b_{l}\left(\nabla \boldsymbol{\phi}_{k}^{\mathrm{T}} \boldsymbol{\varphi}_{l}, \boldsymbol{\varphi}_{i}\right)+\left(\frac{1}{\tau_{p}} \mathbf{I}, \boldsymbol{\varphi}_{i}\right)-\left(\left(\frac{1}{\tau_{p}} \frac{L^{2}-3}{L^{2}-r^{2}}\right) \overline{\boldsymbol{C}}, \boldsymbol{\varphi}_{i}\right)-\sum_{k=1}^{M} b_{k}\left(\left(\frac{1}{\tau_{p}} \frac{L^{2}-3}{L^{2}-r^{2}}\right) \boldsymbol{\varphi}_{k}, \boldsymbol{\varphi}_{i}\right)
\end{aligned}
$$

Finally, we obtain the POD-Galerkin reduced-order model of constitutive equation.

$$
\sum_{k=1}^{M} \frac{d b_{k}}{d t} T 2_{k i}+\sum_{k=1}^{M} \sum a_{k} b_{l} A 2_{k l i}+\sum_{k=1}^{M} a_{k} B 2_{k i}+\sum b_{k} D 2_{k i}=C 2_{i}
$$

Where

$$
\begin{gathered}
T 2_{k i}=\left(\boldsymbol{\varphi}_{k}, \boldsymbol{\varphi}_{i}\right) \\
A 2_{k l i}=\left(\boldsymbol{\phi}_{k} \nabla \boldsymbol{\varphi}_{l}, \boldsymbol{\varphi}_{i}\right)+\left(\nabla \boldsymbol{\phi}_{k} \boldsymbol{\varphi}_{l}, \boldsymbol{\varphi}_{i}\right)-\left(\nabla \boldsymbol{\phi}_{k}^{\mathrm{T}} \boldsymbol{\varphi}_{l}, \boldsymbol{\varphi}_{i}\right) \\
B 2_{k i}=\left(\boldsymbol{\phi} \nabla \overline{\boldsymbol{C}}, \boldsymbol{\varphi}_{i}\right)-\left(\overline{\boldsymbol{C}} \nabla \boldsymbol{\phi}_{k}, \boldsymbol{\varphi}_{i}\right)-\left(\nabla \boldsymbol{\phi}_{k}^{\mathrm{T}} \overline{\boldsymbol{C}}, \boldsymbol{\varphi}_{i}\right) \\
D 2_{k i}=\left(\overline{\boldsymbol{u}} \nabla \boldsymbol{\varphi}_{k}, \boldsymbol{\varphi}_{i}\right)-\left(\boldsymbol{\varphi}_{k} \nabla \overline{\boldsymbol{u}}, \boldsymbol{\varphi}_{i}\right)-\left(\nabla \overline{\boldsymbol{u}}^{\mathrm{T}} \boldsymbol{\varphi}_{k}, \boldsymbol{\varphi}_{i}\right)+\left(\left(\frac{1}{\tau_{p}} \frac{L^{2}-3}{L^{2}-r^{2}}\right) \boldsymbol{\varphi}_{k}, \boldsymbol{\varphi}_{i}\right) \\
C 2_{i}=\left(\overline{\boldsymbol{C}} \nabla \overline{\boldsymbol{u}}, \boldsymbol{\varphi}_{i}\right)-\left(\overline{\boldsymbol{u}} \nabla \overline{\boldsymbol{C}}, \boldsymbol{\varphi}_{i}\right)+\left(\nabla \overline{\boldsymbol{u}}^{\mathrm{T}} \overline{\boldsymbol{C}}, \boldsymbol{\varphi}_{i}\right)+\left(\frac{1}{\tau_{p}} \mathbf{I}, \boldsymbol{\varphi}_{i}\right)-\left(\left(\frac{1}{\tau_{p}} \frac{L^{2}-3}{L^{2}-r^{2}}\right) \overline{\boldsymbol{C}}, \boldsymbol{\varphi}_{i}\right)
\end{gathered}
$$

The combination of Eqs.(20) and (24) gives the POD reduced-order model for the viscoelastic fluid flow. It consists of two series of pluralistic nonlinear equations for $a_{k}$ and $b_{k}$. These equations are coupled and can be solved by a Newton-Raphson method. After $a_{k}$ and $b_{k}$ are obtained, the velocity field and deformation rate can be calculated through Eqs.(14) and (15) and the real velocity field and deformation rate by Eqs.(10) (11).

\section{Results and Discussion}

In this section, the performance of the established reduced order model is systematic studied both on 2-D decaying isotropic turbulence in a square domain (Example 1) and 3-D forced isotropic viscoelastic turbulent flow in cube region (Example 2), which are classical viscoelastic turbulent flow problems. During the implementation of POD reduced order model on these two problems, the basis functions are acquired firstly and the performance of the POD reduced order model is tested subsequently. The computation efficiency of established reduced order model compared with DNS.

\subsection{Basis functions}

To obtain the basis functions, the first step is sampling. In Example 1, the dynamical viscosity is $\mu=5.0 \times 10^{-3} \mathrm{~Pa} \cdot \mathrm{s}$ and it is simulated by DNS using finite volume method on a grid 
system with $64 \times 64$ cells. Three mesh resolutions $64 \times 64,128 \times 128$ and $256 \times 256$, in the $\mathrm{y}$-direction, are considered. The velocity profile in the center line, for different mesh resolutions are shown in Fig. 2 (a). The results revealed that there are no significant differences between the $64 \times 64$ and $128 \times 128$ resolutions. In addition, because of the restrictions of computer memory, the resolution of $64 \times 64$ was chosen for simulation. Then, a set of velocity fields and deformation rate fields are chosen as sampling data to construct the sampling matrices shown. The chosen data are results in every 0.1 second, i.e. $t=\{0.1,0.2,0.3 \ldots 4.8,4.9,5.0\}$. In this way, we obtain 50 sampling cases in total.

For forced isotropic turbulence in Example 2, the flow is directly calculated under $\mu=1.0 \times 10^{-2} \mathrm{~Pa} \cdot \mathrm{s}$ on a gird with $64 \times 64 \times 64$ cells. After comparing the velocity in the center line of z-direction between the $64 \times 64 \times 64$ and $128 \times 128 \times 128$ resolutions shown in Fig. 2 (b), we choose $64 \times 64 \times 64$ resolution to calculate the flow because there are no significant differences between the two resolutions. We choose 100 cases $(t=10.1,10.2,10.3 \ldots 19.8,19.9$, 20.0) of fully developed velocity fields and deformation rate fields to construct matrices.

Secondly, we extract basis functions from sampling matrices. Fig. 3 presents the accumulating spectrum from the POD eigenvalues of the dataset of flow snapshots during the considered time-interval. Fig. 4 shows the energy contribution of each basis function. Furthermore, the specific eigenvalue, energy contribution, and accumulative energy of the first 15 velocity basis functions in Example 1 are given in Table 1. The first velocity basis function contains $61.012 \%$ of the total energy, which is more than the sum energy of the rest basis functions. The second one contains $23.930 \%$ of the total energy, which is much less than the first one does. The latter basis functions contain less and less energy. Combining the above two figures and Table 1, we can find that the convergence rate is quite large and the first 10 modes contain more than $99.9 \%$ of the total kinetic energy in the system. For the deformation rate basis functions, the specific energy information is shown in Table 2. It can be seen that they share the similar rule with the velocity basis functions and the first 15 deformation rate modes contain more than $99.9 \%$ of the total energy.

6 typical contours of the velocity basis functions are given in the Fig.5. It is clearly illustrated that the first basis function corresponds to high-energetic large-scale structures, and the following basis functions correspond to lower-energetic small-scale structures.

According to the practice experience, $99.9 \%$ of the total energy is high enough to get a good and efficient result in most cases. In Example 1, the first 10 velocity basis functions can capture the main characteristics of the turbulence as they occupy most of the energy, while 15 deformation rate basis functions are needed to occupy the $99.9 \%$ of the total energy. Based on this analysis we choose the first 15 basis functions to get an accepted result. The 11th-15th velocity basis functions 
have a very small amount of energy. They are $0.0312 \%, 0.0150 \%, 0.00794 \%, 0.00485 \%$, $0.00258 \%$ respectively. And they represent the small-scale structures because of the small energy contribution. However, they capture the necessary information in local places. This is because energy dissipation takes place at small scales which are required in the reduced-order model.

According to the energy information of velocity basis functions shown in Fig.6 and Fig.7 of Example 2, the energy converges so quickly that only 10 modes contain $99.954 \%$ of the total kinetic energy in the viscoelastic fluid flow system. The specific information of the first 12 velocity basis functions and deformation rate basis functions are given in Table 3 and Table 4 respectively. It is stated that $62.070 \%$ of the total energy is extracted by the first velocity basis function. We choose 12 basis functions to get an accepted result in this example because the first 12 deformation rate basis functions can contain $99.9 \%$ of the total energy. Therefore, it can be concluded that the basis functions of the viscoelastic fluid flow can achieve fast convergence speed or catch flow information quickly. Obviously, this phenomenon is more remarkable in three-dimensional turbulence.

4 typical contours of the velocity basis functions are given in the Fig.8. It is clearly illustrated that the former basis functions correspond to high-energetic large-scale structures, and the latter basis functions correspond to lower-energetic small-scale structures. Since energy dissipation takes place at small scales and we cannot take all these basis functions into account, this will give rise to a significant calculation error inevitably for the complex problem.

\subsection{Test results}

To simplify issues, we only choose one variable parameter (dynamical viscosity) to examine the performance of the studied model in this paper. For the decaying isotropic turbulence, by calculating the spectral coefficients under $\mu=4 \times 10^{-3} \mathrm{~Pa} \cdot \mathrm{s}$ which is different from the samplings', 40 cases differing from the sampling cases for $t=\{0.1,0.2,0.3 \ldots 3.8,3.9,4.0\}$ are obtained to test the accuracy of the proposed reduced-order model in Example 1.

Since our major object is to get velocity field, we focus on velocity spectral coefficients and leave out the deformation rates spectral coefficients here. Fig. 9 presents the spectral coefficients obtained from POD, also gives out the corresponding ones from DNS for comparison. Obviously, the two sets of spectral coefficients are quite similar. When we increased the time to a further stage for example $t=2.0 \mathrm{~s}$, the corresponding spectral coefficients become a little different. Next, Fig. 10 is the phase space of velocity. It is clearly stated that the POD does well in converging compared with DNS for the former ones. When it comes to the fifth spectral coefficient related phase space, the differences become considerable.

In order to quantitatively evaluate the accuracy of the POD reduced-order model, the error of 
velocity is defined as:

$$
e=\left(\sum_{l=1}^{N_{\text {tot }}}\left|u_{P O D}(l)-u_{D N S}(l)\right|\right) /\left(\sum_{l=1}^{N_{\text {tot }}}\left|u_{D N S}(l)\right|\right)
$$

Where, the subscripts "DNS" and "POD" stand for the results calculated by direct numerical simulation and POD reduced-order model respectively.

Errors of the 40 test cases in two examples are shown in Fig. 11 below. It can be clearly seen that the velocity errors are relatively small when the decaying is at its early age. However, as the decaying continues, the errors begin to accumulate. After around $t=0.8 \mathrm{~s}$, the errors are greater than $5 \%$. The reason is that the POD basis functions can capture the main turbulent flow information which corresponds to contain high kinetic energy. Thus a relatively accurate result can be obtained when the decaying is weak and turbulence is strong enough. With the kinetic energy becoming lower and lower, the turbulent flow finally develops into laminar flow through transition of flow pattern. The reason is that the basis functions cannot capture the corresponding flow characteristic because the latter basis functions describe a clutter of small scale vortices. Therefore, the proposed POD reduced-order model cannot depict accurate transition flow. In reference [17], W. Cazemier suggested adding a closure model to the low order model, which can help reduce error of ROM. In his study a linear damping model was used, unfortunately, there is no fixed form for closure modes and the closure models itself are also gotten by try and errors.

The velocity contours obtained by DNS and POD methods are compared at different time instant $(0.1,0.5,1.0,3.0,4.0)$ in Fig. 12. The graphs are the u-velocity results of the studied viscoelastic fluid flow. The relative velocity errors are 1.022\%, 3.754\%, $6.181 \%, 8.458 \%, 8.551 \%$ respectively. It is implied that when the turbulence is just to decay, the turbulent vortex is very small and the errors are insignificant, and while when the turbulent decaying is highly developed, with the vortex becoming greater, the errors getting considerable too, which is illustrated in (e) below.

To examine the performance of the forced isotropic case in Example 2, we set the dynamical viscosity to be $\mu=2.0 \times 10^{-2} \mathrm{~Pa} \cdot \mathrm{s}$ and get 20 cases $(t=10.1,10.2,10.3 \ldots 11.8,11.9,12.0)$ to analyze the results.

In the forced isotropic viscoelastic fluid flow, the velocity errors are small when the solution is at its early age which is shown in Fig.13. However, as the turbulence continues, the errors begin to accumulate. Because turbulence develops erratically over time, the studied method cannot predict it for a long time. However, the errors of viscoelastic forced isotropic turbulent flow are acceptable for application.

To get a more straightforward understanding of the performance, the velocity contours of viscoelastic fluid obtained by DNS and POD methods are compared at different time instant (10.1, 
10.5, 11.0, 12.0) in Fig.14. Since the three-dimensional contour is not an intuitive way to observe the accuracy, we project the three-dimensional results on two-dimensional image planes ( $\mathrm{x}-\mathrm{y}, \mathrm{y}-\mathrm{z}$, $\mathrm{x}-\mathrm{y})$ respectively. It is implied that the results are quite accurate at the beginning of the solution, and gradually the results become more and more inaccurate by increasing the calculation step.

\subsection{Computation efficiency}

A comparison of the computational time cost by DNS and POD method is considered in this part. The computational time cost of two cases is shown in Table.5 and Table.6, respectively. Obviously, the advantage of the ROM is remarkable with its computation speed of the ROM being tens of times faster than that of DNS in Example1, and this advantage would be much more remarkable on further calculation. In Example2, the ROM and DNS both require relatively more time, which results from the large quantity of computation time to calculate equation coefficients in ROM and the deformation equation in DNS. Actually, in POD, the computational time is mainly spent on coefficients of the model and varies little with the increase of calculation time step. Whereas, the time cost by DNS show a linear positive correlation with time step. We can safely conclude that the studied method has distinct advantages in calculation speed.

\section{Conclusions}

The POD-Galerkin reduced-order model for the turbulent flow of viscoelastic fluid is established and systematically evaluated in this paper for the first time. Through the reduced-order model, the fast calculations of both the decaying and forced isotropic turbulent flows under different parameters can be realized. Two corresponding examples are given in this study to illustrate the performance of the proposed reduced-order model. For the decaying isotropic turbulence, it is found that the established reduced-order model has good accuracy which is less than $5 \%$ when the decaying flow is at its early stage, but the errors become considerable when the flow develops into transition flow. For the forced isotropic turbulence, it is illustrated that the errors of the studied viscoelastic fluid flow model are quite small at the beginning of the solution. However, the errors get accumulated with time going by. The established POD reduced order model has practical significance because the error is acceptable to some extent and it is also a necessary study for further study on POD reduced order model study on viscoelastic turbulent flow in the future.

\section{Acknowledgement}

This work was supported by the National Natural Science Foundation of China (51476073) and the National Science Foundation for Distinguished Young Scholars of China (51325603).

\section{References}


[1] R.J. Adrian, Conditional eddies in isotropic turbulence, Physics of Fluids. 22 (1979) 2065-2070.

[2] J.L. Lumley, The structure of inhomogeneous turbulent flows, Atmospheric Turbulence and Radio Wave Propagation. 1967 166-178.

[3] H.P. Bakewell, J.L. Lumley, Viscous sublayer and adjacent wall region in turbulent pipe flow, The Physics of Fluids. 10 (1967) 1880-1889.

[4] S. Herzog, The large scale structure in the near-wall region of a turbulent pipe flow, $\mathrm{PhD}$ thesis, Cornell University. 1986.

[5] D. Rempfer, On the structure of dynamical systems describing the evolution of coherent structures in a convective boundary layer, Physics of Fluids. 6 (1994) 1402-1404.

[6] D. Rempfer, H.F. Fasel, Evolution of three-dimensional coherent structures in a flat-plate boundary layer, Journal of Fluid Mechanics. 260 (1994) 351-375.

[7] N. Aubry, P. Holmes, J.L. Lumley, et al, The dynamics of coherent structures in the wall region of a turbulent boundary layer, Journal of Fluid Mechanics. 192 (1988) 115-173.

[8] P.J. Holmes, J.L. Lumley, G. Berkooz, et al, Low-dimensional models of coherent structures in turbulence, Physics Reports. 287 (1997) 337-384.

[9] P.S. Johansson, H.I. Andersson, E.M. Ronquist, Reduced-basis modeling of turbulent plane channel flow, Computers \& fluids. 35 (2006) 189-207.

[10] P.S. Johansson, H.I. Andersson, Generation of inflow data for inhomogeneous turbulence, Theoretical and Computational Fluid Dynamics. 18 (2004) 371-389.

[11] S. Rahal, P. Cerisier, H. Azuma, Application of the proper orthogonal decomposition to turbulent convective flows in a simulated Czochralski system, International Journal of Heat and Mass Transfer. 51 (2008) 4216-4227.

[12] B. Podvin, A proper-orthogonal-decomposition-based model for the wall layer of a turbulent channel flow, Physics of Fluids. 21 (2009) 115.

[13] L. Sirovich, Turbulence and the dynamics of coherent structures. Part 1: Coherent structures, Quarterly of applied mathematics. 45 (1987) 561-590.

[14] V.M. Tikhomirov, Local structure of turbulence in an incompressible viscous fluid at very large Reynolds numbers, Selected Works of A. N. Kolmogorov, Springer Netherlands. 1991 9-13.

[15] G. Samanta, A.N. Beris, R.A. Handler, et al, Velocity and conformation statistics based on reduced Karhunen-Loeve projection data from DNS of viscoelastic turbulent channel flow, Journal of Non-Newtonian Fluid Mechanics. 160 (2009) 55-63.

[16] G. Samanta, K.D. Housiadas, A.N. Beris, et al, Data reduction in viscoelastic turbulent channel flows based on extended Karhunen-Loeve analysis, Journal of Non-Newtonian Fluid Mechanics. 165 (2010) 1386-1399. 
[17] W. Cazemier, R.W.C.P. Verstappen, A.E.P. Veldman, Proper orthogonal decomposition and low-dimensional models for driven cavity flows, Phys. Fluids. 10 (1998) 1685-1699.

[18] L. Kourentis, E. Konstantinidis, Uncovering large-scale coherent structures in natural and forced turbulent wakes by combining PIV, POD, and FTLE, Experiments in Fluids. 52 (2012) 749-763.

[19] A. Orellano, H. Wengle, POD analysis of coherent structures in forced turbulent flow over a fence, Journal of Turbulence. 2 (2001) 8-8(1).

[20] W. Cazemier, Proper orthogonal decomposition and low dimensional models for turbulent flows, Groningen. 1997.

[21] S.Y. Motlagh, S. Taghizadeh, POD analysis of low Reynolds turbulent porous channel flow, International Journal of Heat and Fluid Flow. 61 (2016) 665-676.

[22] D. Greig, K. Siddiqui, P. Karava, A. Elatar, Investigation of fundamental flow mechanisms over a corrugated waveform using proper orthogonal decomposition and spectral analyses, International Journal of Thermal Sciences. 96 (2015) 160-172.

[23] D. Stein, B. Thomases, POD analysis of viscoelastic flow instabilities, APS Division of Fluid Dynamics Meeting Abstracts. 2014. 


\section{Table captions:}

Table 1 Energy contribution of the first 15 velocity basis functions for Example 1

Table 2 Energy contribution of the first 15 deformation rate basis functions for Example 1

Table 3 Energy contribution of the first 12 velocity basis functions for Example 2

Table 4 Energy contribution of the first 12 deformation rate basis functions for Example 2

Table 5 Comparison of computational time cost by POD-ROM and DNS in Example 1

Table 6 Comparison of computational time cost by POD-ROM and DNS in Example 2 


\section{Figure captions:}

Fig. 1 Sampling matrices construction

Fig. 2 Profiles of u-velocity in the center line for different grids

Fig. 3 Accumulative energy of the velocity basis functions in Example 1

Fig. 4 Energy contribution of the velocity basis functions in Example 1

Fig. 5 Contours of $\phi_{k u}$ for Example 1

Fig. 6 Accumulative energy of the velocity basis functions in Example 2.

Fig. 7 Energy contribution of the velocity basis functions in Example 2

Fig. 8 Contours of $\phi_{k u}$ for Example 2 (left: $\mathrm{x}-\mathrm{y}$, right: $\mathrm{y}-\mathrm{z}$ )

Fig. 9 Spectral coefficients obtained from POD and DNS

Fig. 10 the Comparison between velocity phase space of POD and DNS

Fig. 11 Relative error of velocity by POD in Example 1

Fig. 12 Comparison of the contours by DNS and POD in Example 1

Fig. 13 Relative error of velocity by POD in Example 2

Fig. 14 Comparison of the u-contours by DNS and POD in Example 2 
Table 1 Energy contribution of the first 15 velocity basis functions for Example 1

\begin{tabular}{|c|c|c|c|c|c|}
\hline$n$ & 1 & 2 & 3 & 4 & 5 \\
\hline$\lambda_{\mathrm{n}}$ & $2.59 \times 10^{4}$ & $1.02 \times 10^{4}$ & $3.71 \times 10^{3}$ & $1.39 \times 10^{3}$ & $6.04 \times 10^{2}$ \\
\hline$\varsigma_{\mathrm{n}}$ & $61.012 \%$ & $23.930 \%$ & $8.718 \%$ & $3.278 \%$ & $1.420 \%$ \\
\hline$\varpi_{\mathrm{n}}$ & $61.012 \%$ & $84.942 \%$ & $93.660 \%$ & $96.937 \%$ & $98.357 \%$ \\
\hline$n$ & 6 & 7 & 8 & 9 & 10 \\
\hline$\lambda_{\mathrm{n}}$ & $3.55 \times 10^{2}$ & $1.65 \times 10^{2}$ & $8.55 \times 10$ & $4.15 \times 10$ & $2.37 \times 10$ \\
\hline$\varsigma_{\mathrm{n}}$ & $0.835 \%$ & $0.388 \%$ & $0.201 \%$ & $0.0975 \%$ & $0.0557 \%$ \\
\hline$\varpi_{\mathrm{n}}$ & $99.193 \%$ & $99.581 \%$ & $99.782 \%$ & $99.879 \%$ & $99.935 \%$ \\
\hline$n$ & 11 & 12 & 13 & 14 & 15 \\
\hline$\lambda_{\mathrm{n}}$ & $1.33 \times 10$ & 6.39 & 3.38 & 2.06 & 1.10 \\
\hline$\varsigma_{\mathrm{n}}$ & $0.0312 \%$ & $0.0150 \%$ & $0.00794 \%$ & $0.00485 \%$ & $0.00258 \%$ \\
\hline$\varpi_{\mathrm{n}}$ & $99.966 \%$ & $99.981 \%$ & $99.989 \%$ & $99.994 \%$ & $99.997 \%$ \\
\hline
\end{tabular}


Table 2 Energy contribution of the first 15 deformation rate basis functions for Example 1

\begin{tabular}{llllll}
\hline$n$ & 1 & 2 & 3 & 4 & 5 \\
\hline \hline$\lambda_{\mathrm{n}}$ & $8.33 \times 10^{3}$ & $3.34 \times 10^{3}$ & $1.51 \times 10^{3}$ & $8.70 \times 10^{2}$ & $4.39 \times 10^{2}$ \\
$\varsigma_{\mathrm{n}}$ & $54.512 \%$ & $21.826 \%$ & $9.866 \%$ & $5.692 \%$ & $2.873 \%$ \\
$\varpi_{\mathrm{n}}$ & $54.512 \%$ & $76.339 \%$ & $86.205 \%$ & $91.897 \%$ & $94.770 \%$ \\
\hline \multicolumn{7}{c}{} & & & & \\
\hline \hline$\lambda_{\mathrm{n}}$ & 6 & 7 & 8 & 9 & 10 \\
$\varsigma_{\mathrm{n}}$ & $3.08 \times 10^{2}$ & $1.75 \times 10^{2}$ & $1.25 \times 10^{2}$ & $6.85 \times 10$ & $4.59 \times 10$ \\
$\varpi_{\mathrm{n}}$ & $2.019 \%$ & $1.144 \%$ & $0.815 \%$ & $0.448 \%$ & $0.301 \%$ \\
\hline & $96.789 \%$ & $97.933 \%$ & $98.748 \%$ & $99.196 \%$ & $99.496 \%$ \\
\hline \hline$\lambda_{\mathrm{n}}$ & & & & & \\
$\varsigma_{\mathrm{n}}$ & $2.80 \times 10$ & $1.90 \times 10$ & $1.05 \times 10$ & 7.44 & 4.44 \\
$\varpi_{\mathrm{n}}$ & $0.183 \%$ & $0.124 \%$ & $0.0688 \%$ & $0.0488 \%$ & $0.0290 \%$ \\
\hline
\end{tabular}


Table 3 Energy contribution of the first 12 velocity basis functions for Example 2

\begin{tabular}{ccccccc}
\hline$n$ & 1 & 2 & 3 & 4 & 5 & 6 \\
\hline \hline$\lambda_{\mathrm{n}}$ & $2.03 \times 10^{4}$ & $7.79 \times 10^{3}$ & $2.71 \times 10^{3}$ & $1.06 \times 10^{3}$ & $4.48 \times 10^{2}$ & $5.01 \times 10^{3}$ \\
$\varsigma_{\mathrm{n}}$ & $62.070 \%$ & $23.839 \%$ & $8.292 \%$ & $3.241 \%$ & $1.371 \%$ & $0.605 \%$ \\
$\varpi_{\mathrm{n}}$ & $62.070 \%$ & $85.909 \%$ & $94.201 \%$ & $97.442 \%$ & $98.814 \%$ & $99.418 \%$ \\
\hline$n$ & 7 & 8 & 9 & & 10 & 11 \\
\hline$\lambda_{\mathrm{n}}$ & $9.53 \times 10$ & $4.50 \times 10$ & $2.28 \times 10$ & $1.21 \times 10$ & $6.44 \times 10$ & $3.64 \times 10$ \\
$\varsigma_{\mathrm{n}}$ & $0.292 \%$ & $0.138 \%$ & $0.0698 \%$ & $0.0370 \%$ & $0.0197 \%$ & $0.0114 \%$ \\
$\varpi_{\mathrm{n}}$ & $99.710 \%$ & $99.847 \%$ & $99.917 \%$ & $99.954 \%$ & $99.974 \%$ & $99.985 \%$ \\
\hline
\end{tabular}


Table 4 Energy contribution of the first 12 deformation rate basis functions for Example 2

\begin{tabular}{ccccccc}
\hline$n$ & 1 & 2 & 3 & 4 & 5 & 6 \\
\hline \hline$\lambda_{\mathrm{n}}$ & $1.11 \times 10^{5}$ & $5.54 \times 10^{4}$ & $2.77 \times 10^{4}$ & $1.63 \times 10^{4}$ & $8.61 \times 10^{3}$ & $5.01 \times 10^{3}$ \\
$\varsigma_{\mathrm{n}}$ & $47.859 \%$ & $23.993 \%$ & $11.992 \%$ & $7.055 \%$ & $3.727 \%$ & $2.169 \%$ \\
$\varpi_{\mathrm{n}}$ & $47.859 \%$ & $71.852 \%$ & $83.844 \%$ & $90.898 \%$ & $94.625 \%$ & $96.794 \%$ \\
\hline$n$ & 7 & 8 & 9 & & 10 & 12 \\
\hline \hline$\lambda_{\mathrm{n}}$ & $2.92 \times 10^{3}$ & $1.74 \times 10^{3}$ & $1.03 \times 10^{3}$ & $6.32 \times 10^{2}$ & $3.94 \times 10^{2}$ & $2.50 \times 10^{2}$ \\
$S_{\mathrm{n}}$ & $1.263 \%$ & $0.754 \%$ & $0.446 \%$ & $0.274 \%$ & $0.230 \%$ & $0.138 \%$ \\
$\varpi_{\mathrm{n}}$ & $98.057 \%$ & $98.811 \%$ & $99.257 \%$ & $99.530 \%$ & $99.761 \%$ & $99.899 \%$ \\
\hline
\end{tabular}


Table 5 Comparison of computational time cost by POD-ROM and DNS in Example 1

\begin{tabular}{cccc}
\hline $\mathrm{t}$ & $\mathrm{POD}(\mathrm{S})$ & $\mathrm{DNS}(\mathrm{s})$ & Multiple \\
1 & 2.79 & 44.06 & 15.8 \\
2 & 2.90 & 88.13 & 30.4 \\
3 & 2.92 & 128.02 & 43.8 \\
4 & 3.00 & 156.82 & 52.3 \\
\hline
\end{tabular}


Table 6 Comparison of computational time cost by POD-ROM and DNS in Example 2

\begin{tabular}{cccc}
\hline $\mathrm{t}$ & $\mathrm{POD}(\mathrm{S})$ & $\mathrm{DNS}(\mathrm{s})$ & Multiple \\
11.0 & 110.2 & 500.2 & 4.54 \\
12.0 & 112.3 & 998.3 & 8.89 \\
13.0 & 113.4 & 1398.2 & 12.33 \\
\hline
\end{tabular}




$$
\left[\begin{array}{c}
u^{\prime}\left(x_{1}, y_{1}, z_{1}, i\right) \\
\mathrm{M} \\
u^{\prime}\left(x_{p}, y_{q}, z_{l}, i\right) \\
v^{\prime}\left(x_{1}, y_{1}, z_{1}, i\right) \\
\mathrm{M} \\
v^{\prime}\left(x_{p}, y_{q}, z_{l}, i\right) \\
w^{\prime}\left(x_{1}, y_{1}, z_{1}, i\right) \\
\mathrm{M} \\
w^{\prime}\left(x_{p}, y_{q}, z_{l}, i\right)
\end{array}\right] \Rightarrow\left[\begin{array}{ccccc}
u^{\prime}\left(x_{1}, y_{1}, z_{1}, 1\right) & \ldots & u^{\prime}\left(x_{1}, y_{1}, z_{1}, i\right) & \ldots & u^{\prime}\left(x_{1}, y_{1}, z_{1}, N\right) \\
\mathrm{M} & & & & \\
u^{\prime}\left(x_{p}, y_{q}, z_{l}, 1\right) & \ldots & u^{\prime}\left(x_{p}, y_{q}, z_{l}, i\right) & \ldots & u^{\prime}\left(x_{p}, y_{q}, z_{l}, N\right) \\
v^{\prime}\left(x_{1}, y_{1}, z_{1}, 1\right) & \ldots & v^{\prime}\left(x_{1}, y_{1}, z_{1}, i\right) & \ldots & v^{\prime}\left(x_{1}, y_{1}, z_{1}, N\right) \\
\mathrm{M} & & & & \\
v^{\prime}\left(x_{p}, y_{q}, z_{l}, 1\right) & \ldots & v^{\prime}\left(x_{p}, y_{q}, z_{l}, i\right) & \ldots & v^{\prime}\left(x_{p}, y_{q}, z_{l}, N\right) \\
w^{\prime}\left(x_{1}, y_{1}, z_{1}, 1\right) & \ldots & w^{\prime}\left(x_{1}, y_{1}, z_{1}, i\right) & \ldots & w^{\prime}\left(x_{1}, y_{1}, z_{1}, N\right) \\
\mathrm{M} & & & & \\
w^{\prime}\left(x_{p}, y_{q}, z_{l}, 1\right) & \ldots & w^{\prime}\left(x_{p}, y_{q}, z_{l}, i\right) & \ldots & w^{\prime}\left(x_{p}, y_{q}, z_{l}, N\right)
\end{array}\right]
$$

(a) Velocity sampling matrix construction

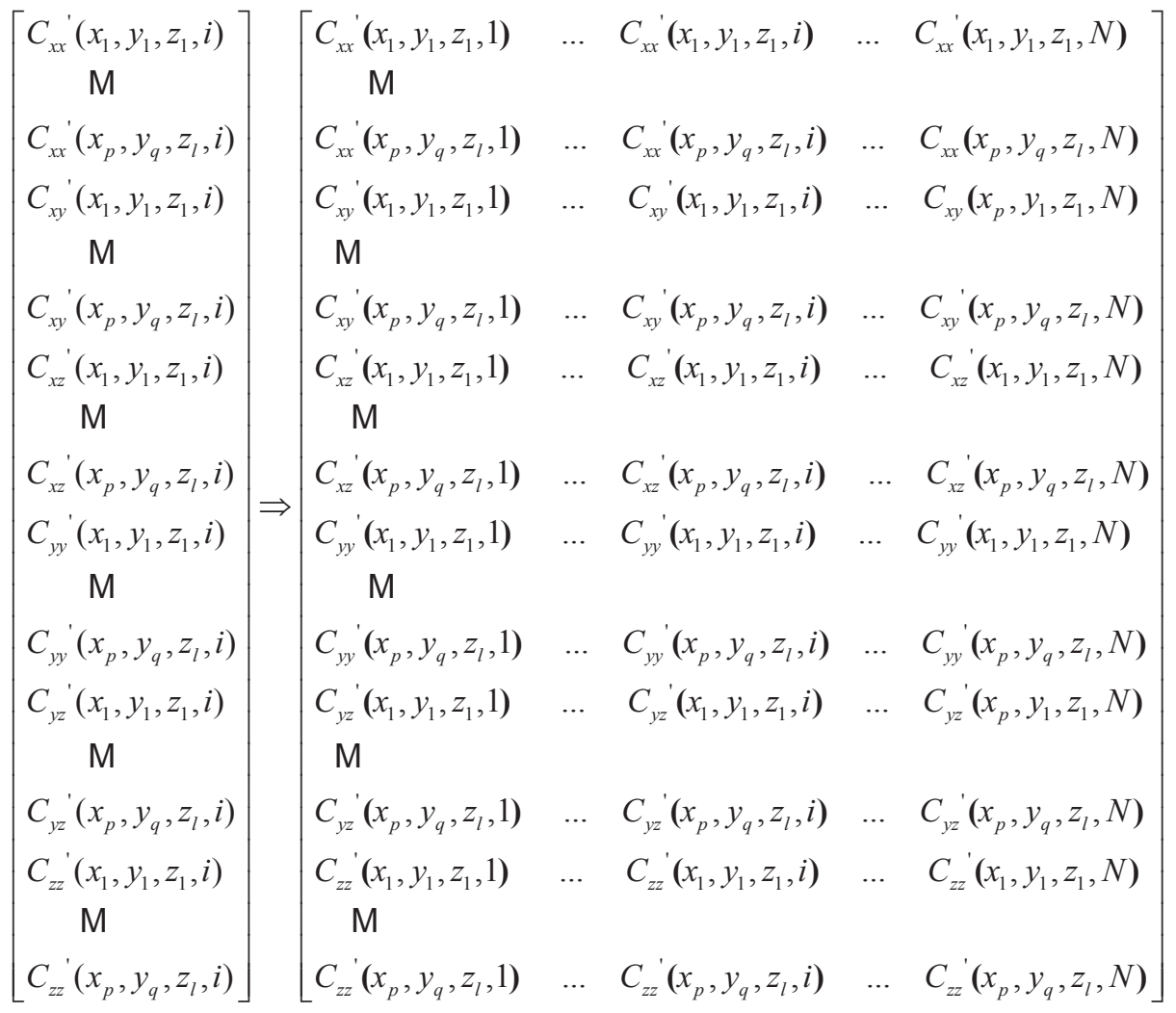

(b) Deformation rate sampling matrix construction

Fig. 1 Sampling matrices construction 


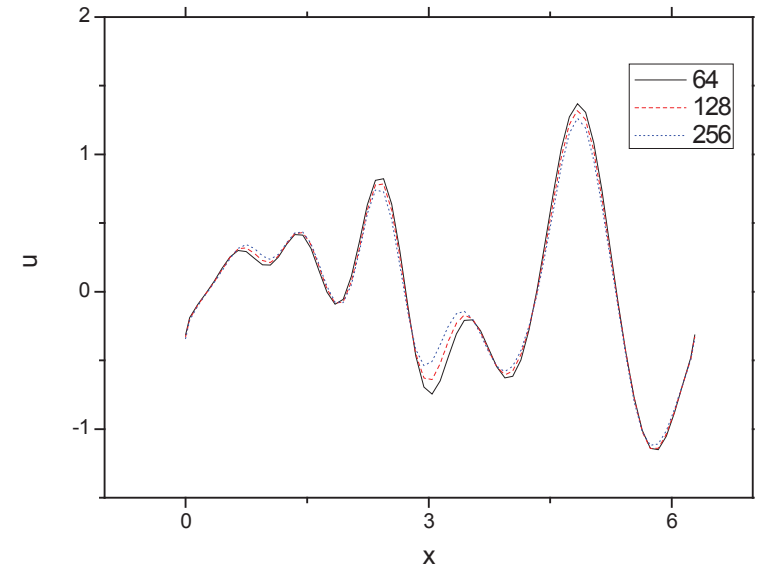

(a) Profiles of wall-normal u-velocity in Example 1

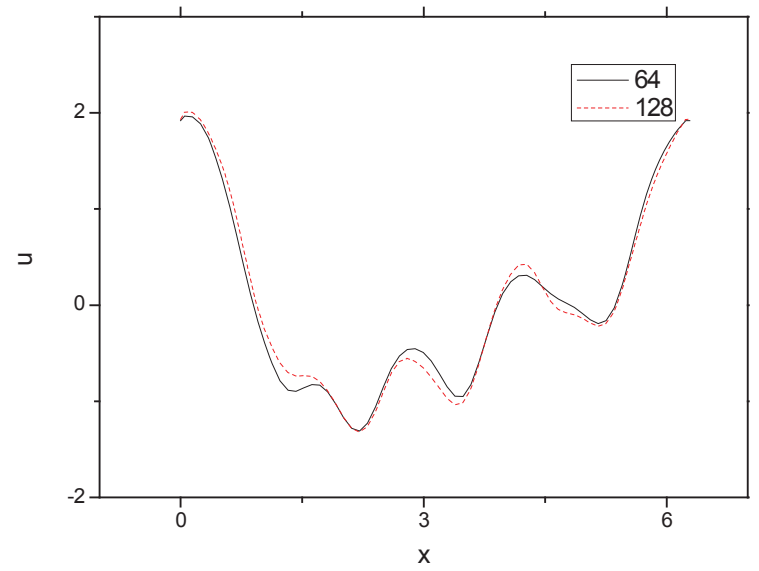

(b) Profiles of span-wise u-velocity in Example 2

Fig. 2 Profiles of u-velocity in the center line for different grids 


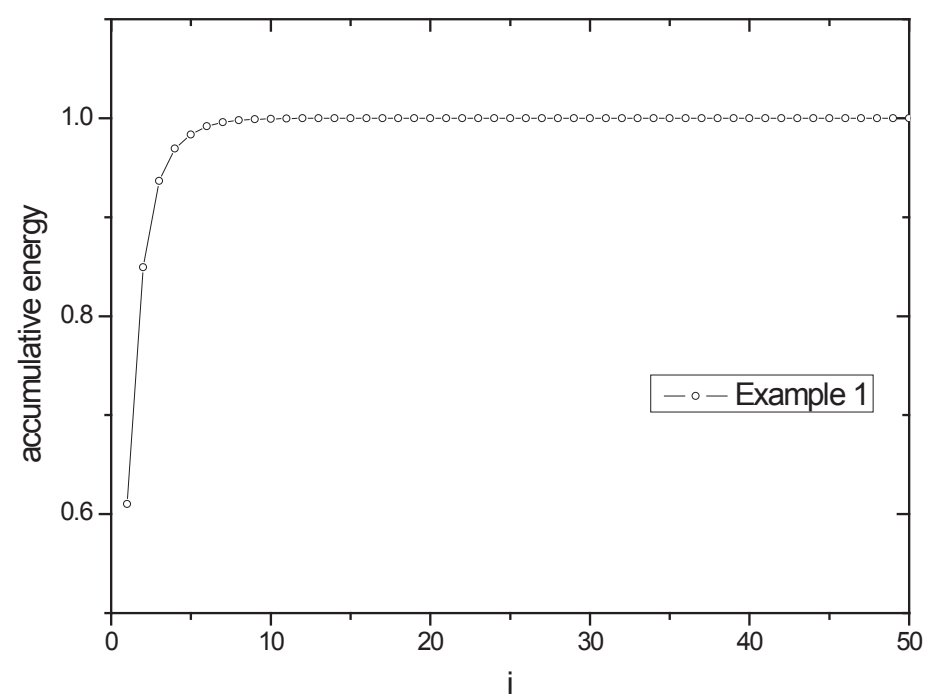

Fig. 3 Accumulative energy of the velocity basis functions in Example 1. 


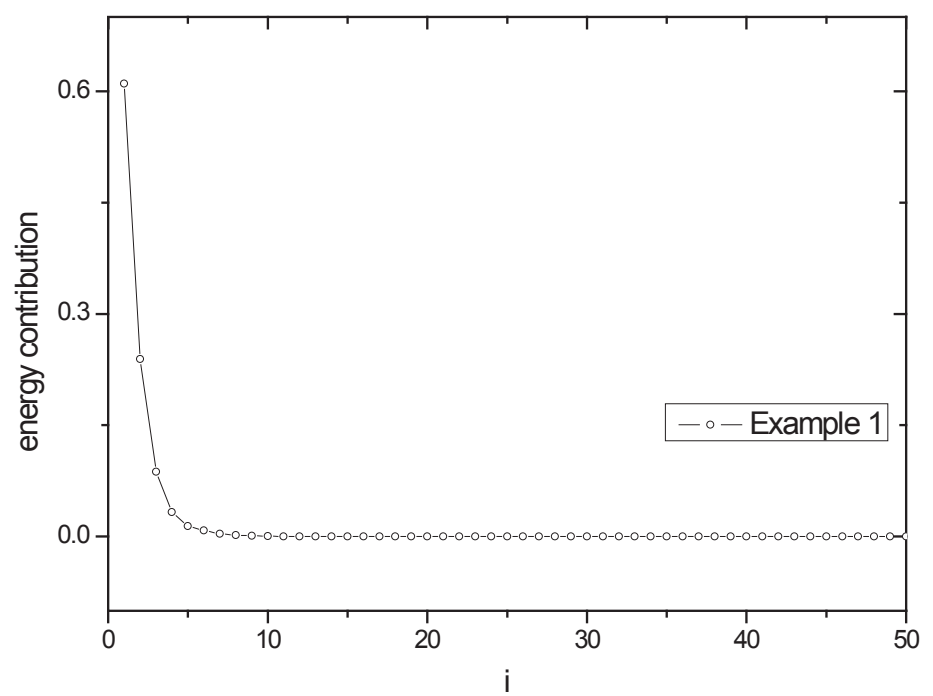

Fig. 4 Energy contribution of the velocity basis functions in Example 1. 

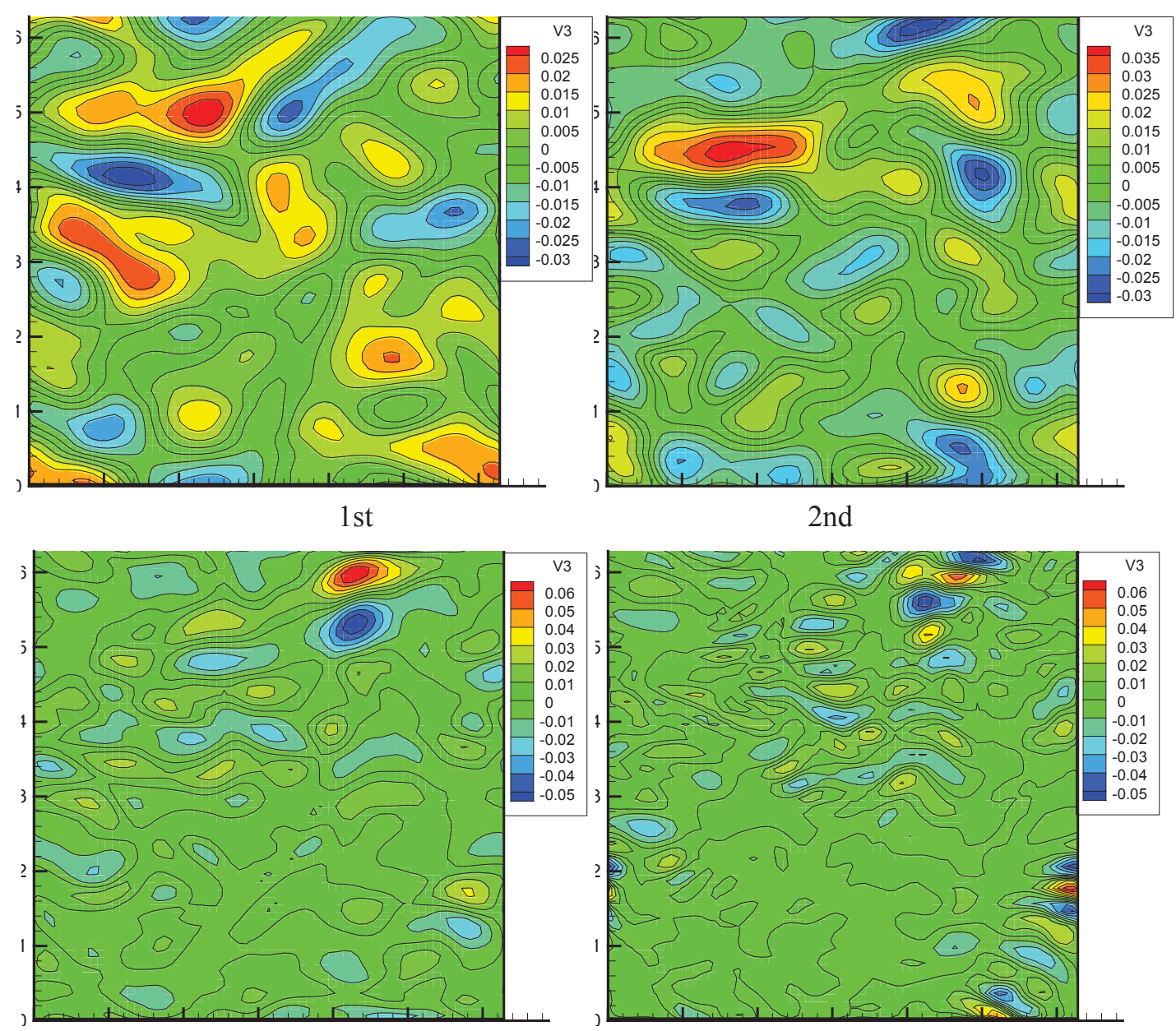

5 th

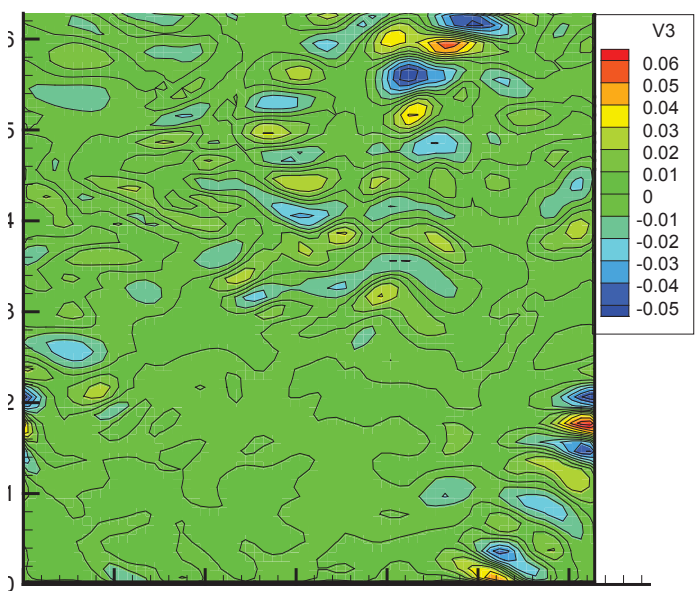

10th

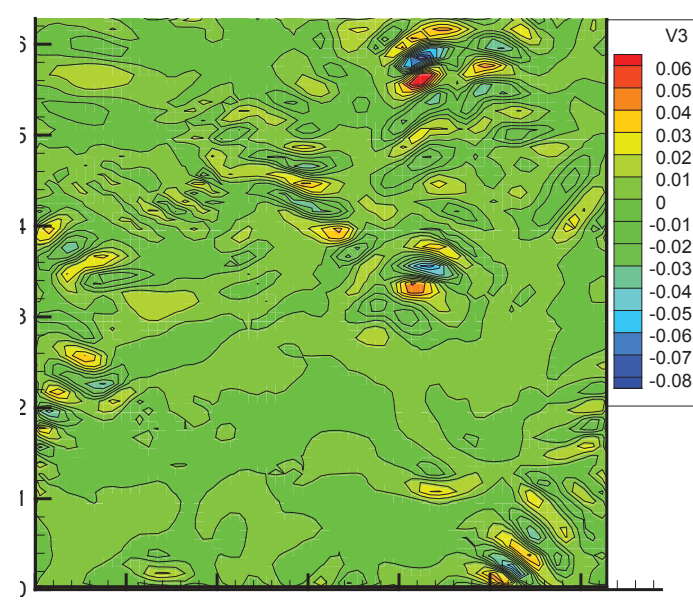

15 th

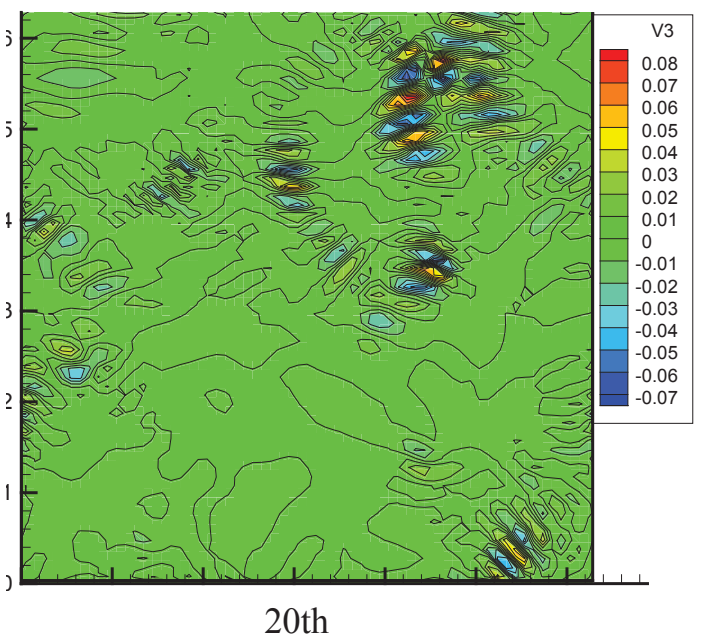

Fig. 5 Contours of $\phi_{k u}$ for Example 1 


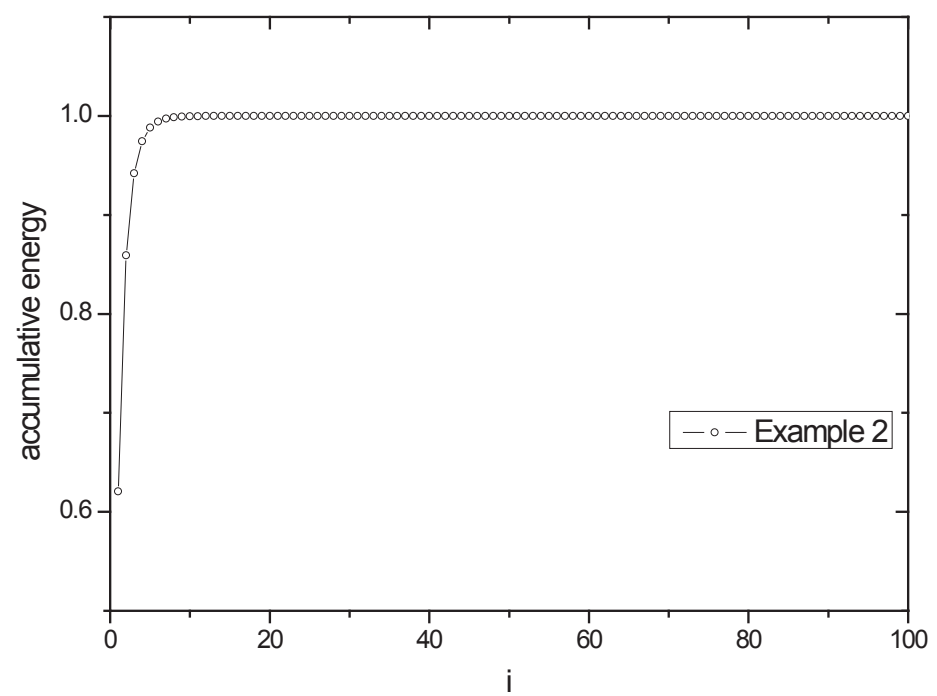

Fig. 6 Accumulative energy of the velocity basis functions in Example 2. 


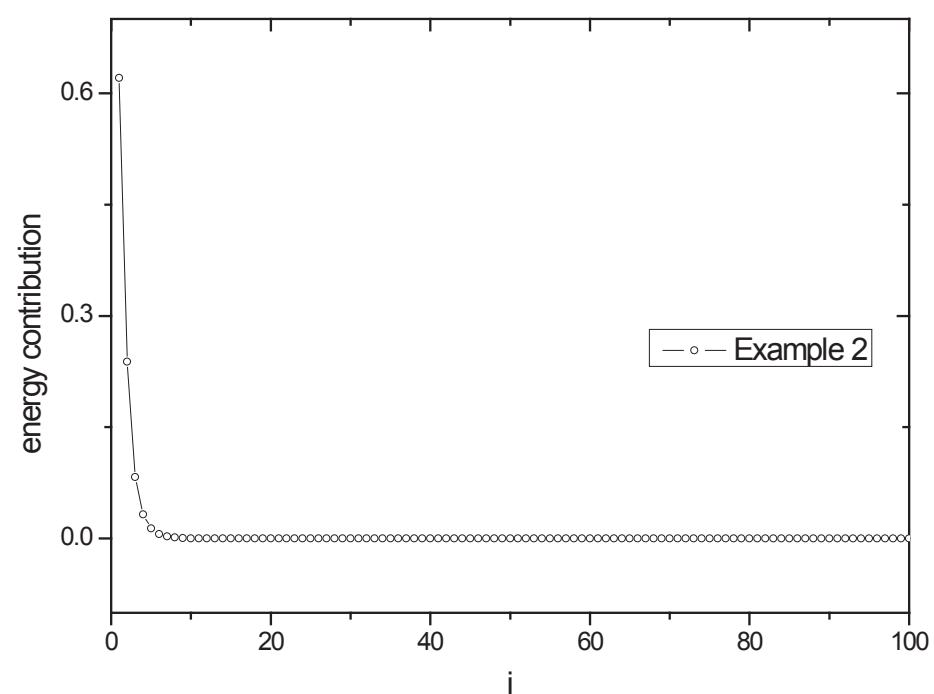

Fig. 7 Energy contribution of the velocity basis functions in Example 2 

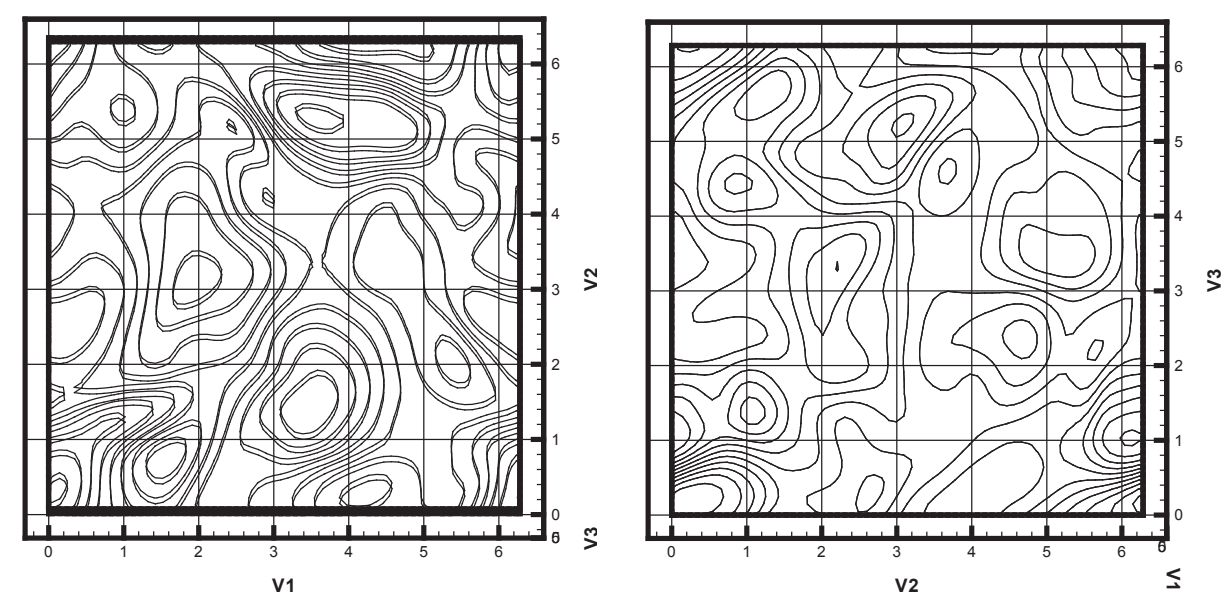

$1 \mathrm{st}$
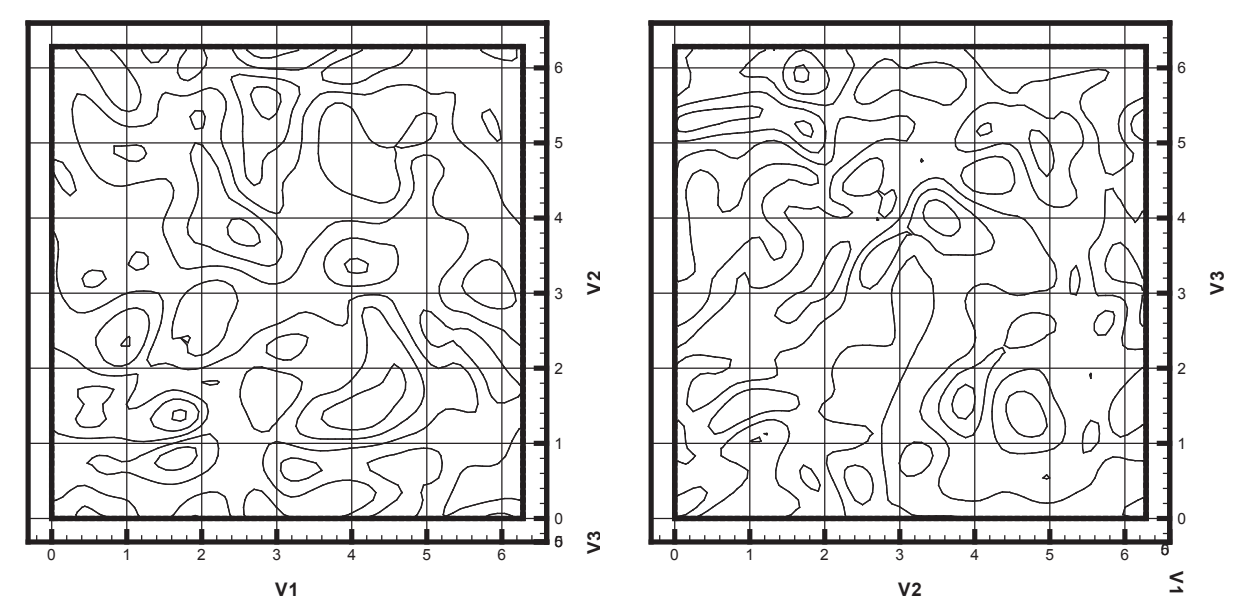

5th
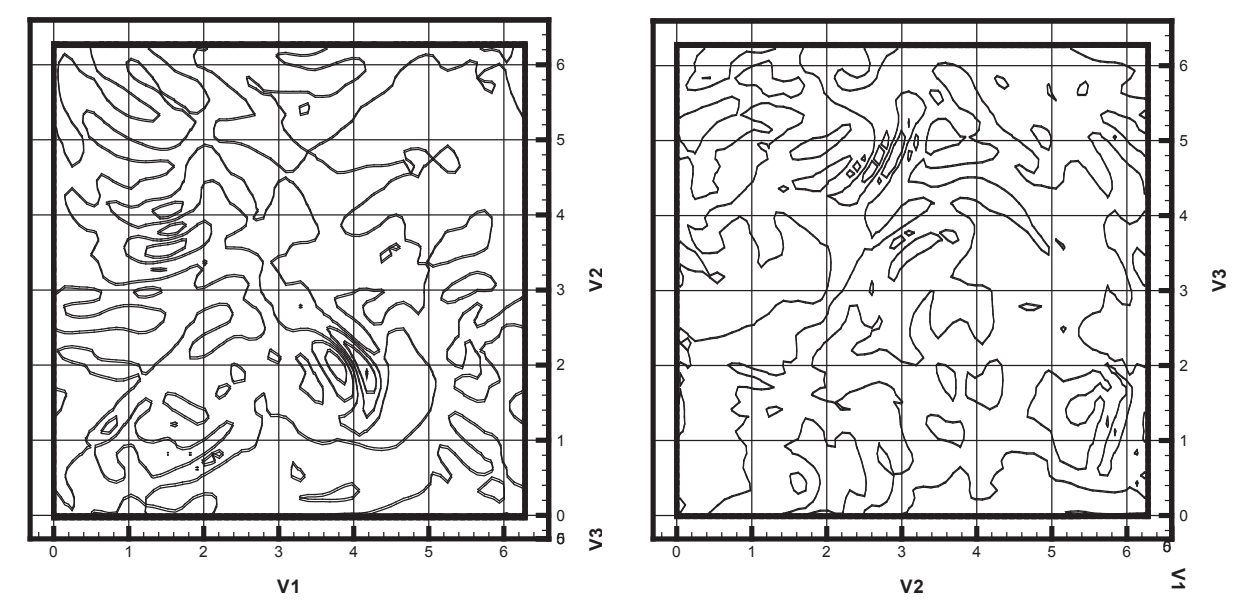

20th 

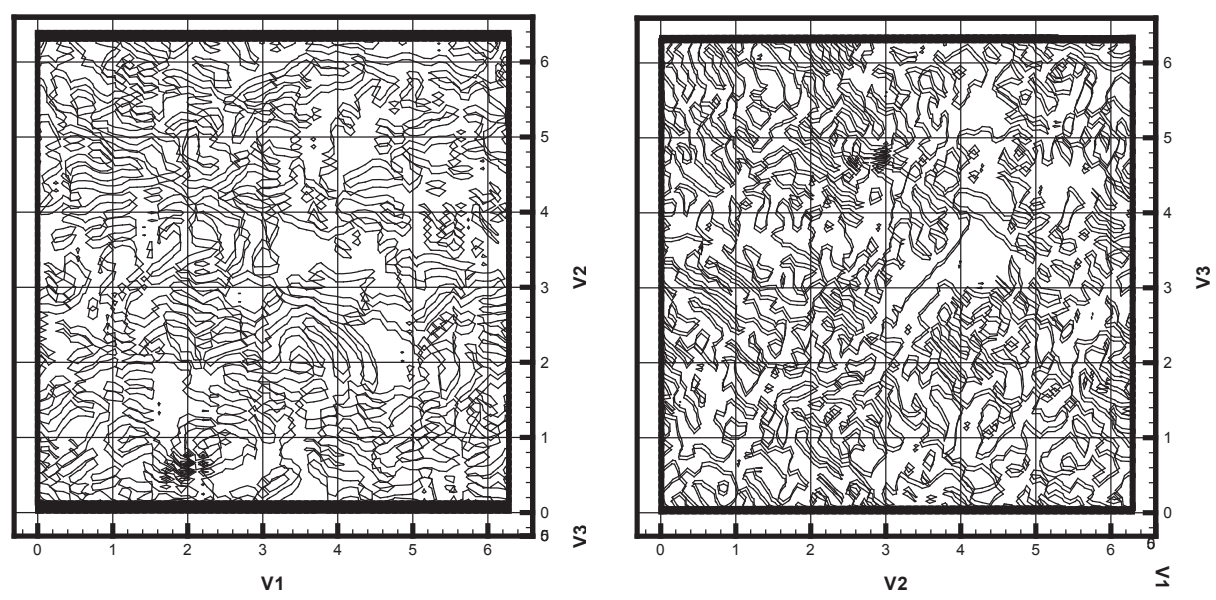

50 th

Fig. 8 Contours of $\phi_{k u}$ for Example 2 (left: x-y, right: y-z) 


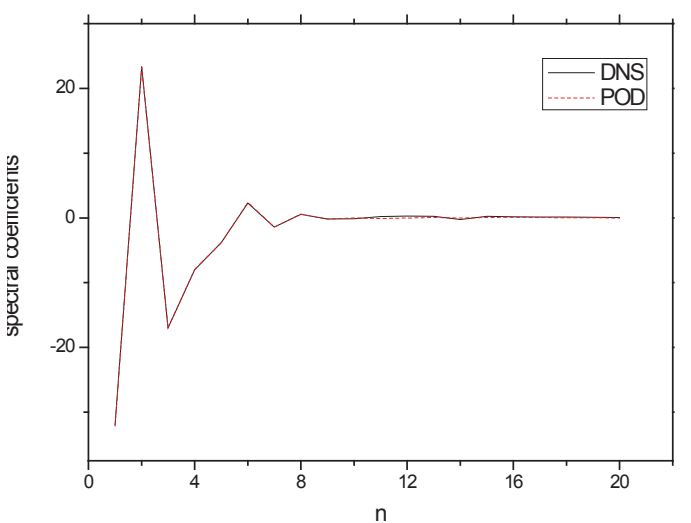

(a) $t=0.1 \mathrm{~s}$

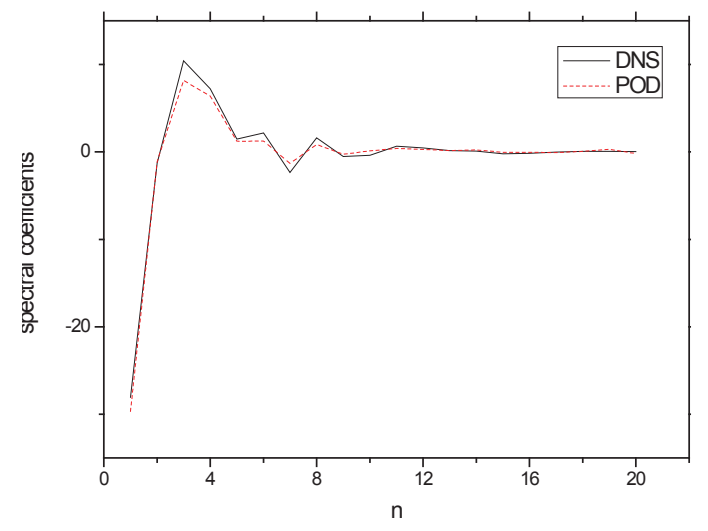

(c) $t=1.0 \mathrm{~s}$

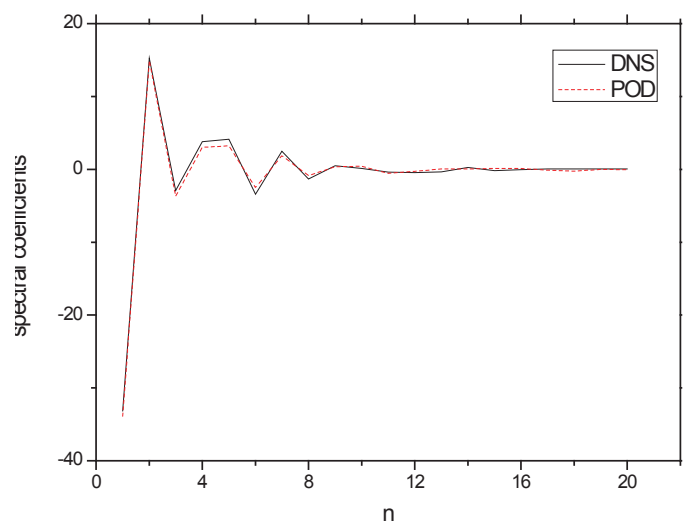

(b) $t=0.5 \mathrm{~s}$

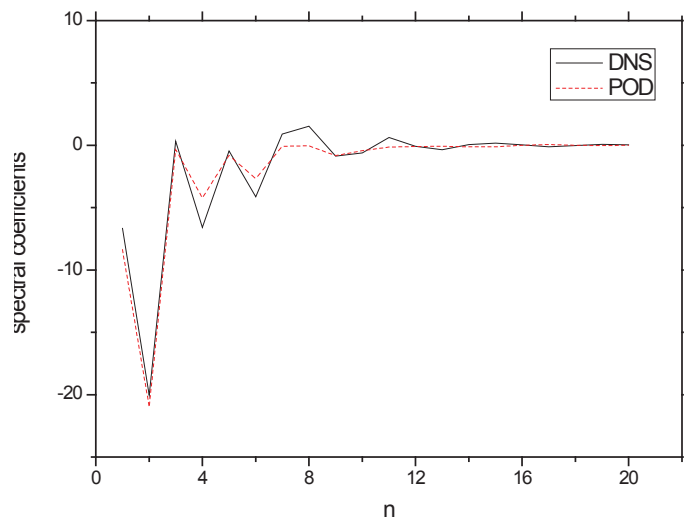

(d) $t=2.0 \mathrm{~s}$

Fig. 9 Spectral coefficients obtained from POD and DNS 


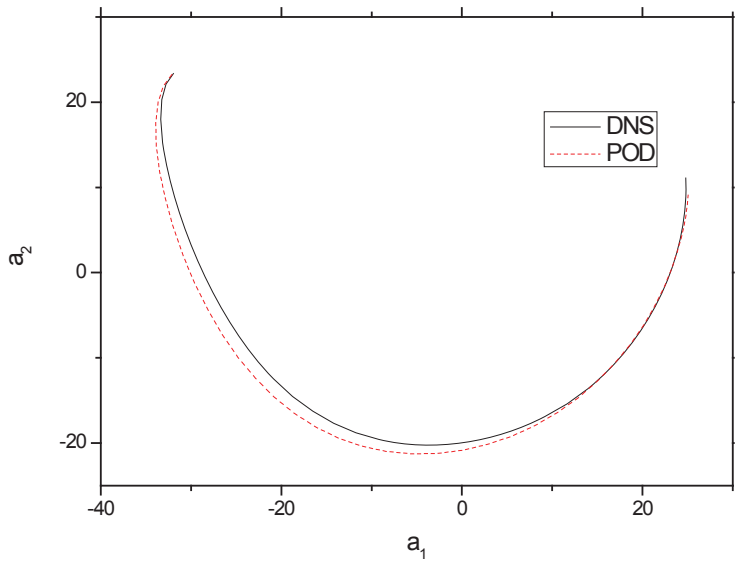

(a) $a_{1}-a_{2}$

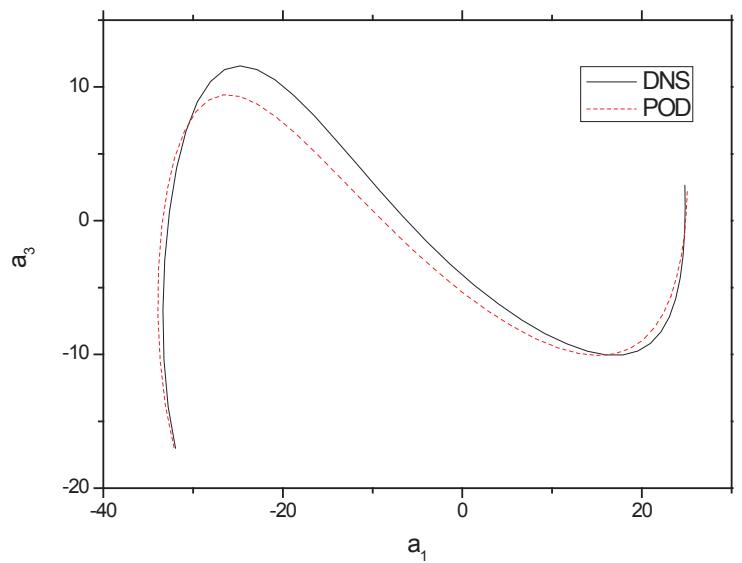

(b) $\mathrm{a}_{1}-\mathrm{a}_{3}$

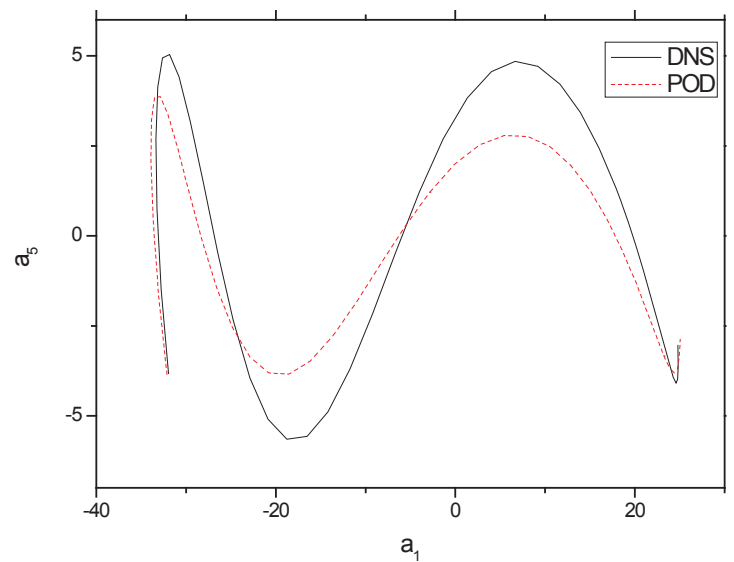

(c) a1-a5

Fig. 10 the Comparison between velocity phase space of POD and DNS 


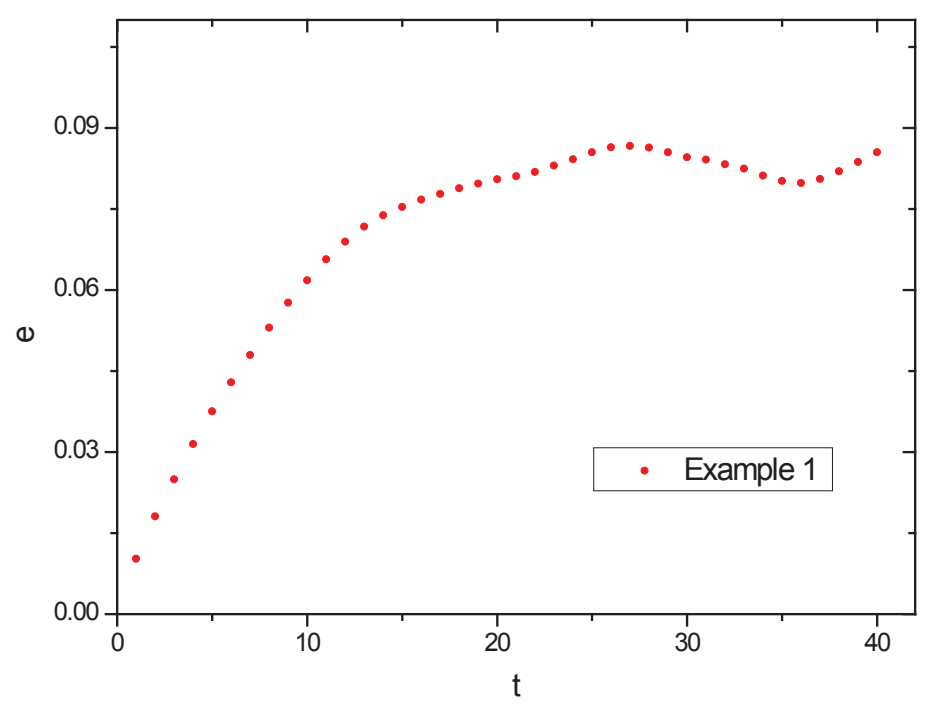

Fig. 11 Relative error of velocity by POD in Example 1 


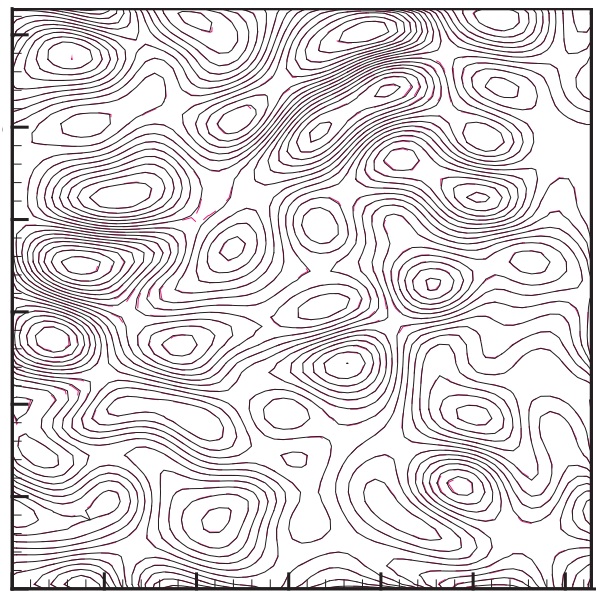

(a) $t=0.1 \mathrm{~s}(\mathrm{e}=1.022 \%)$

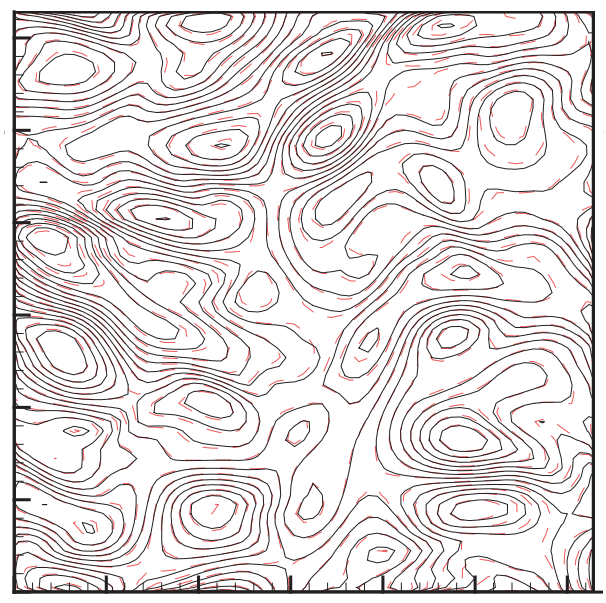

(c) $t=1.0 \mathrm{~s}(\mathrm{e}=6.181 \%)$

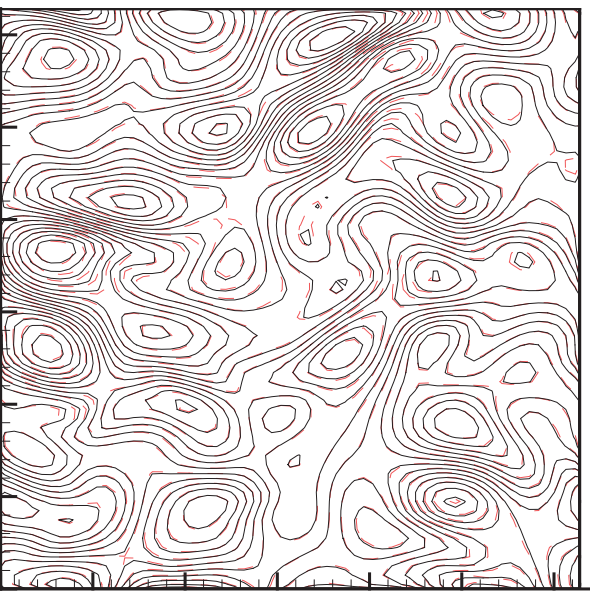

(b) $t=0.5 \mathrm{~s}(\mathrm{e}=3.754 \%)$

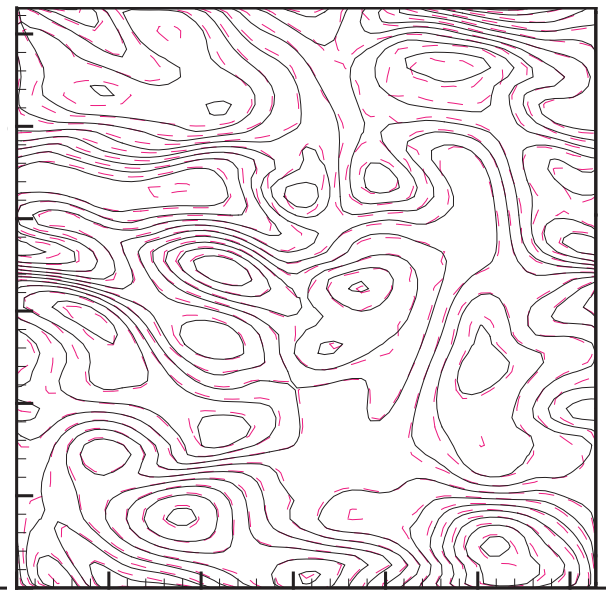

(d) $t=3.0 \mathrm{~s}(\mathrm{e}=8.458 \%)$

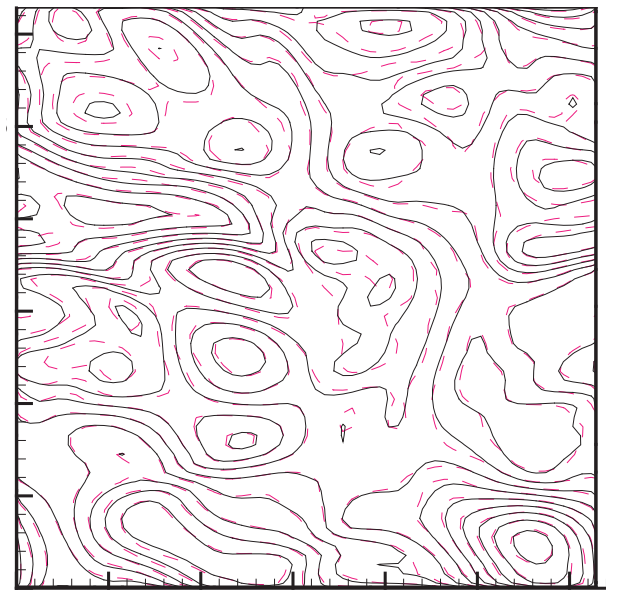

(e) $t=4.0 \mathrm{~s}(\mathrm{e}=8.551 \%)$

(Black solid line: DNS, Red dashed line: POD)

Fig. 12 Comparison of the contours by DNS and POD in Example 1 


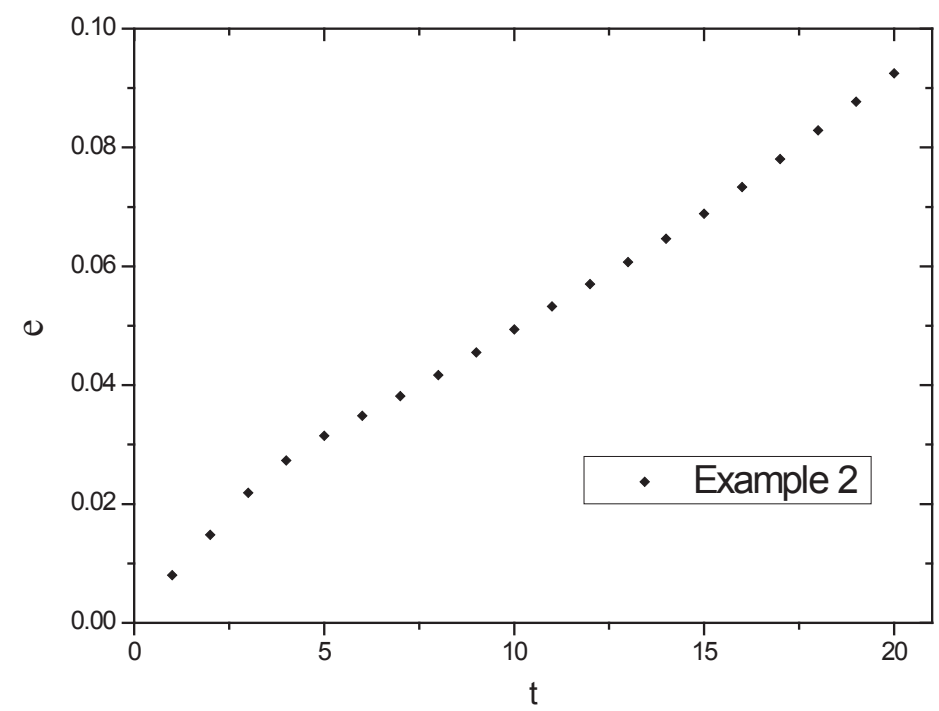

Fig. 13 Relative error of velocity by POD in Example 2 

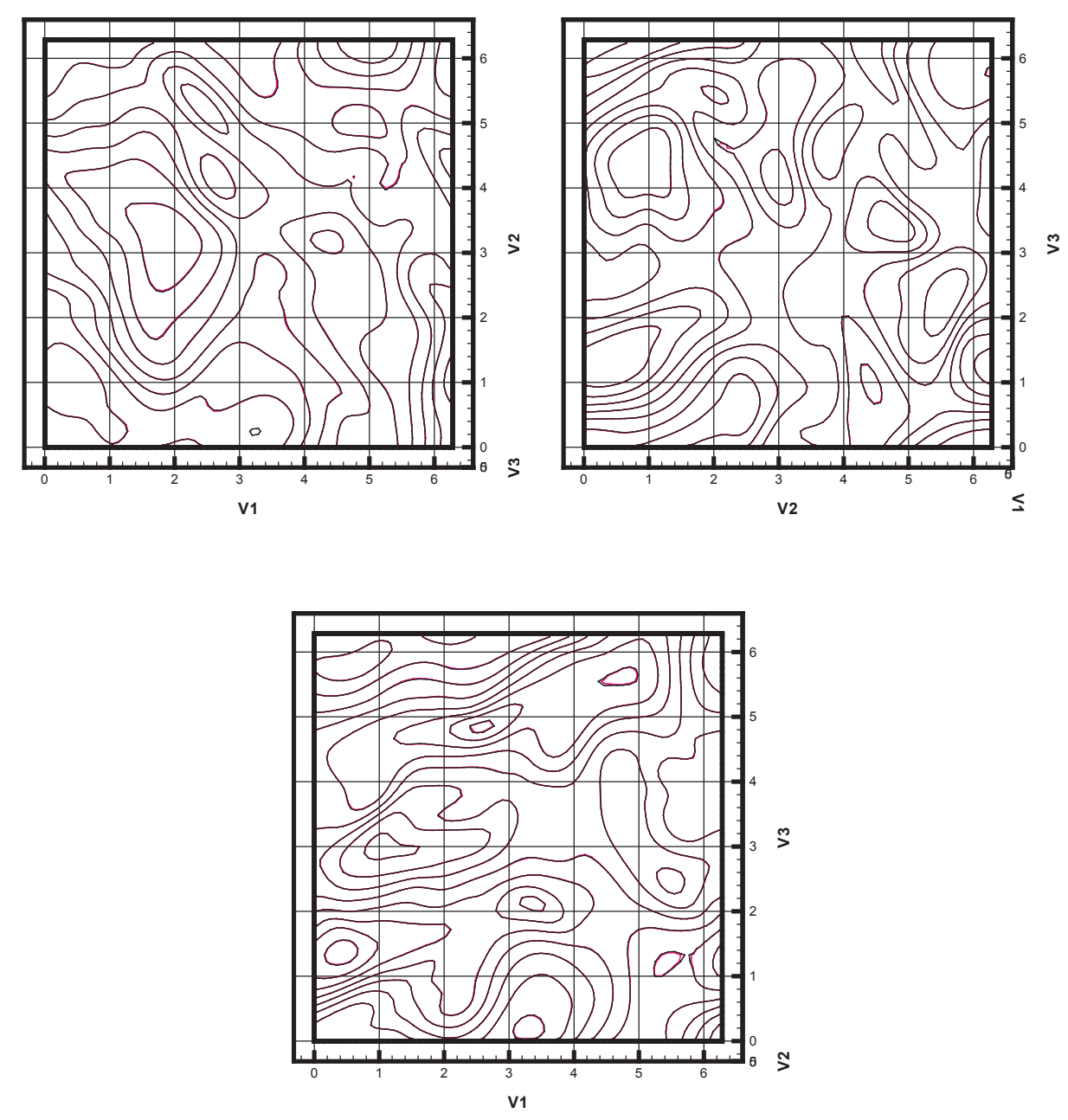

(a) $t=10.1 \mathrm{~s}(\mathrm{e}=0.802 \%)$
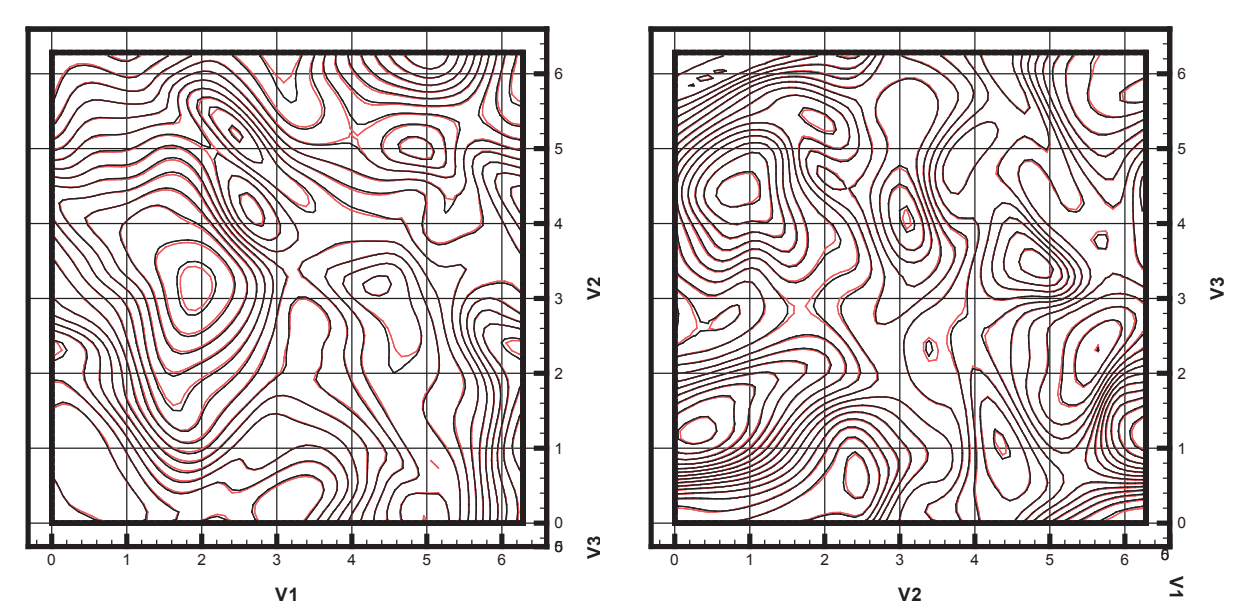


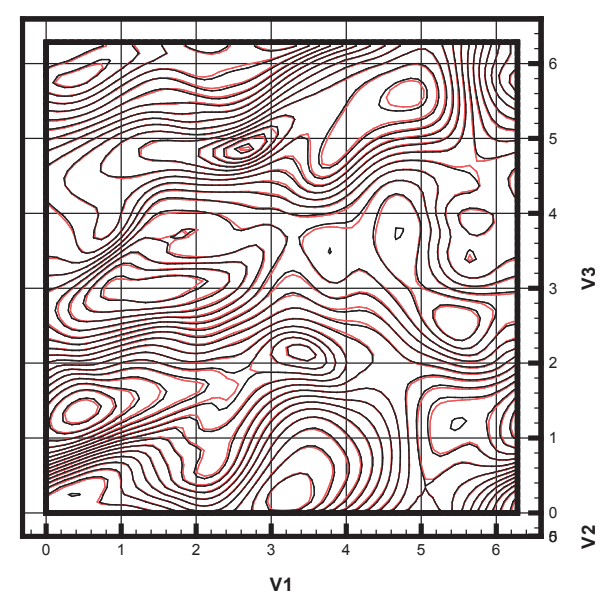

(b) $t=10.5 \mathrm{~s}(\mathrm{e}=3.145 \%)$
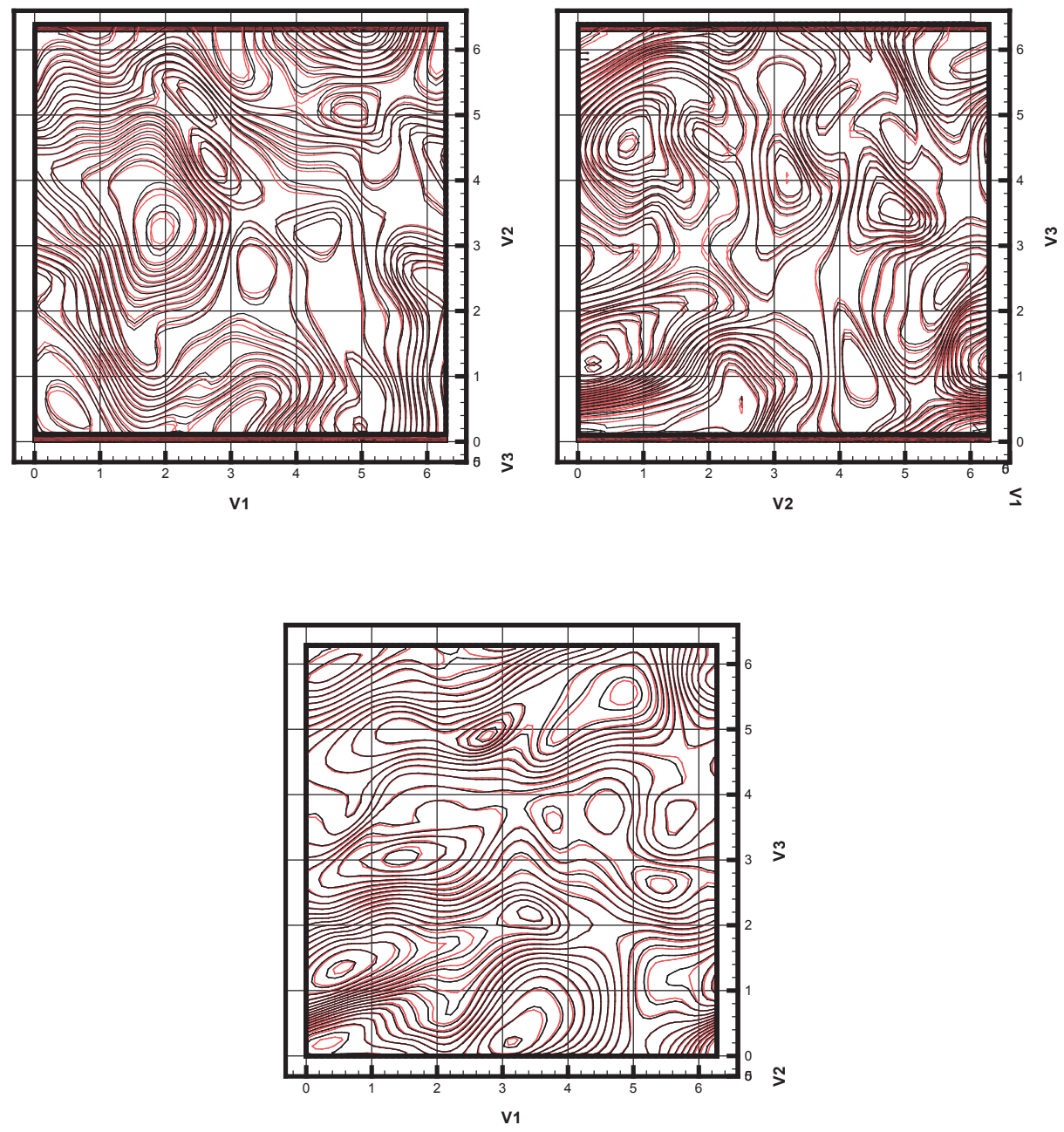

(c) $t=11.0 \mathrm{~s}(\mathrm{e}=4.940 \%)$ 